\title{
Dynamic Behaviors of a Discrete Lotka-Volterra Competition System with Infinite Delays and Single Feedback Control
}

\author{
Liang Zhao, ${ }^{1}$ Xiangdong Xie, ${ }^{2}$ Liya Yang, ${ }^{1}$ and Fengde Chen ${ }^{1}$ \\ ${ }^{1}$ College of Mathematics and Computer Science, Fuzhou University, Fuzhou, Fujian 350116, China \\ ${ }^{2}$ Department of Mathematics, Ningde Normal University, Fujian 352100, China
}

Correspondence should be addressed to Xiangdong Xie; ndsyxxd@163.com

Received 21 May 2014; Revised 28 July 2014; Accepted 28 July 2014; Published 14 October 2014

Academic Editor: Ming Mei

Copyright (c) 2014 Liang Zhao et al. This is an open access article distributed under the Creative Commons Attribution License, which permits unrestricted use, distribution, and reproduction in any medium, provided the original work is properly cited.

\begin{abstract}
A nonautonomous discrete two-species Lotka-Volterra competition system with infinite delays and single feedback control is considered in this paper. By applying the discrete comparison theorem, a set of sufficient conditions which guarantee the permanence of the system is obtained. Also, by constructing some suitable discrete Lyapunov functionals, some sufficient conditions for the global attractivity and extinction of the system are obtained. It is shown that if the the discrete Lotka-Volterra competitive system with infinite delays and without feedback control is permanent, then, by choosing some suitable feedback control variable, the permanent species will be driven to extinction. That is, the feedback control variable, which represents the biological control or some harvesting procedure, is the unstable factor of the system. Such a finding overturns the previous scholars' recognition on feedback control variables.
\end{abstract}

\section{Introduction}

During the last decade, the study of the dynamic behaviors of discrete time models governed by difference equation has become one of the most important topics in mathematics biology; many interesting results concerned with permanence, extinction, and existence of positive periodic solution (almost periodic solution) and so forth have been extensively studied by many scholars; see [1-30] and the references cited therein.

As far as two-species discrete competition model is concerned, Chen and Zhou [1] proposed and studied the following discrete two-species Lotka-Volterra system:

$$
\begin{aligned}
& x_{1}(n+1) \\
& \quad=x_{1}(n) \exp \left[r_{1}(n)\left(1-\frac{x_{1}(n)}{K_{1}(n)}-\mu_{2}(n) x_{2}(n)\right)\right], \\
& x_{2}(n+1) \\
& \quad=x_{2}(n) \exp \left[r_{2}(n)\left(1-\mu_{1}(n) x_{1}(n)-\frac{x_{2}(n)}{K_{2}(n)}\right)\right],
\end{aligned}
$$

where $K_{i}(n), i=1,2$ represent the environmental carrying capacity of species $x_{1}$ and $x_{2}$, respectively; $r_{i}(n), i=1,2$ are the intrinsic growth rate of two species; and $x_{1}(n)$, $x_{2}(n)$ represent the density of species $x_{1}$ and $x_{2}$ at the $n$th generation, respectively. The authors obtained a set of sufficient conditions which ensure the persistence of system (1). Also, for the periodic case, they gave a set of sufficient conditions which guarantee the existence of a globally stable periodic solution of the system. Chen [2] argued that it is more realistic to incorporate delays into system (1), and he proposed and investigated the following model:

$$
\begin{aligned}
x_{1}(n+1)=x_{1}(n) \exp \left\{a_{1}(n)-b_{11}(n) x_{1}(n)-b_{12}(n)\right. \\
\left.\times \sum_{s=0}^{\infty} k_{12}(s) x_{2}(n-s)\right\}, \\
x_{2}(n+1)=x_{2}(n) \exp \left\{a_{2}(n)-b_{22}(n) x_{2}(n)-b_{21}(n)\right. \\
\left.\times \sum_{s=0}^{\infty} k_{21}(s) x_{1}(n-s)\right\} .
\end{aligned}
$$


Concerned with the persistent property of system (2), the author obtained the following result.

Throughout this paper, given a bounded nonnegative sequence $f(n)$ defined on $Z$, let $f^{L}$ and $f^{M}$ denote inf ${ }_{n \in Z} f(n)$ and $\sup _{n \in Z} f(n)$, respectively.

Theorem A. Assume that

$$
\frac{b_{11}^{L}}{b_{21}^{M}}>\frac{\exp \left(a_{1}^{M}-1\right)}{a_{2}^{L}}, \quad \frac{a_{1}^{L}}{\exp \left(a_{2}^{M}-1\right)}>\frac{b_{12}^{M}}{b_{22}^{L}}
$$

hold; then system (2) is permanent.

However, the author did not investigate the stability and extinction property of the system (2), which are two of the most important topics on the study of population dynamics.

On the other hand, it is well known that, in the real world, ecosystems are continuously disturbed by unpredictable forces which can result in some changes of the biological parameters such as survival rates ([3]). For having a more accurate description of such a system, scholars introduced feedback controls into ecosystems and studied a variety of systems with feedback controls. Based on the work of Chen and Zhou [1], X. X. Chen and F. D. Chen [4] proposed and studied the following nonautonomous two-species discrete competitive system with feedback controls:

$$
\begin{aligned}
& x_{1}(n+1)=x_{1}(n) \exp \left\{r _ { 1 } ( n ) \left[1-\frac{x_{1}(n)}{K_{1}(n)}-\mu_{2}(n) x_{2}(n)\right.\right. \\
&\left.\left.-b_{1}(n) u_{1}(n)\right]\right\}, \\
& x_{2}(n+1)\left.\left.-\frac{x_{2}(n)}{K_{2}(n)}-b_{2}(n) u_{2}(n)\right]\right\}, \\
&=x_{2}(n) \exp \left\{r _ { 2 } ( n ) \left[1-\mu_{1}(n) x_{1}(n)\right.\right. \\
& u_{1}(n+1)=u_{1}(n)\left(1-a_{1}(n)\right)+c_{1}(n) x_{1}(n), \\
& u_{2}(n+1)=u_{2}(n)\left(1-a_{2}(n)\right)+c_{2}(n) x_{2}(n) .
\end{aligned}
$$

Some sufficient conditions for the persistence and global stability of system (3) were obtained. $\mathrm{Xu}$ et al. [5] further considered the following two-species nonautonomous LotkaVolterra competitive system with delays and feedback controls:

$$
\begin{aligned}
& X_{1}(n+1) \\
& =X_{1}(n) \exp \left\{a_{1}(n)-b_{11}(n)\right. \\
& \quad \times \sum_{s=0}^{\tau_{11}} k_{11}(s) X_{1}(n-s)-b_{12}(n)
\end{aligned}
$$

$$
\begin{aligned}
& \times \sum_{s=0}^{\tau_{12}} k_{12}(s) X_{2}(n-s)-d_{1}(n) \\
& \left.\times \sum_{s=0}^{\delta_{1}} h_{1}(s) u_{1}(n-s)\right\}
\end{aligned}
$$

$X_{2}(n+1)$

$$
=X_{2}(n) \exp \left\{a_{2}(n)-b_{22}(n)\right.
$$$$
\times \sum_{s=0}^{\tau_{22}} k_{11}(s) X_{2}(n-s)-b_{21}(n)
$$$$
\times \sum_{s=0}^{\tau_{21}} k_{21}(s) X_{1}(n-s)-d_{2}(n)
$$$$
\left.\times \sum_{s=0}^{\delta_{2}} h_{2}(s) u_{2}(n-s)\right\} \text {, }
$$

$$
\begin{aligned}
& u_{1}(n+1) \\
& \quad=u_{1}(n)\left(1-e_{1}(n)\right)+c_{1}(n) \sum_{s=0}^{\sigma_{1}} l_{1}(s) X_{1}(n-s), \\
& u_{2}(n+1) \\
& \quad=u_{2}(n)\left(1-e_{2}(n)\right)+c_{2}(n) \sum_{s=0}^{\sigma_{2}} l_{2}(s) X_{2}(n-s) .
\end{aligned}
$$

By using the comparison theorem of discrete differential equation and constructing a suitable discrete type Lyapunov functional, they obtained new sufficient conditions on the permanence of species and global attractivity for system (4). Their results show that feedback controls are harmless to the permanence of system (4); that is, feedback controls have no influence on the permanence of system (4). X. Chen and F. Chen [29] and Liao et al. [30] also proposed a discrete time periodic $n$-species Lotka-Volterra competition system with feedback controls and deviating arguments; some sufficient conditions which ensure the existence of unique globally asymptotically stable periodic solution were obtained. Recently, Wu and Zhang [19] proposed a discrete autonomous Lotka-Volterra competition system with infinite delays and feedback controls; by using the iterative method, sufficient conditions which ensure the global attractivity of the system were obtained.

As we can see, those models considered in $[4,5,19,29,30]$ contain two or more feedback control variables, which means that, for the different species, different control strategy is adopted. But, in the real world, the strategy adopted for one species may also affect the other species; in other words, such a strategy has influence on both species. For instance, in the agricultural system, spraying pesticide can reduce the number of weeds, but pesticide can also have a negative impact on the growth of crops or beneficial animals $[6,7]$. 
In the medical system, when doctor takes chemotherapeutic drugs as tools to cure the cancer patients, cancer cells will decrease rapidly, but at the same time, drugs also do harm to normal cells and body's regulatory immune function. Yao et al. [8] studied the effect of chemotherapeutic drugs on cellular immunity in patients with lung cancer; they found that cell immunity is inhibited in patients with lung cancer; moreover, it is impaired considerably by chemotherapy. So how to keep the negative effect caused by the single strategy adopted for the weeds or cancer cells to a minimum?

The above phenomenons motivated us to propose and study the discrete Lotka-Volterra competition system with infinite delays and single feedback control variable as follows:

$$
\begin{aligned}
& x_{1}(n+1) \\
& =x_{1}(n) \exp \left\{a_{1}(n)-b_{11}(n) x_{1}(n)-b_{12}(n)\right. \\
& \times \sum_{s=0}^{\infty} k_{12}(s) x_{2}(n-s)-d_{1}(n) \\
& \left.\times \sum_{s=0}^{\infty} h_{1}(s) u(n-s)\right\} \text {, } \\
& x_{2}(n+1) \\
& =x_{2}(n) \exp \left\{a_{2}(n)-b_{22}(n) x_{2}(n)-b_{21}(n)\right. \\
& \times \sum_{s=0}^{\infty} k_{21}(s) x_{1}(n-s)-d_{2}(n) \\
& \left.\times \sum_{s=0}^{\infty} h_{2}(s) u(n-s)\right\} \text {, } \\
& u(n+1) \\
& =u(n)(1-e(n))+c_{1}(n) \\
& \times \sum_{s=0}^{\infty} l_{1}(s) x_{1}(n-s)+c_{2}(n) \\
& \times \sum_{s=0}^{\infty} l_{2}(s) x_{2}(n-s) \text {. }
\end{aligned}
$$

In system (5), $x_{i}(n)(i=1,2)$ is the density of $x_{i}$ species at the $n$th generation and $u(n)$ is the single feedback control variable.

Throughout this paper, we assume the following.

$\left(\mathrm{H}_{1}\right) a_{i}(n), b_{i j}(n), c_{i}(n), d_{i}(n)$, and $e_{i}(n)(i, j=1,2)$ are bounded sequences of real numbers defined on $Z$ such that

$$
\begin{array}{r}
a_{i}^{L}>0, \quad b_{i j}^{L}>0, \quad c_{i}^{L}>0, \\
d_{i}^{L}>0, \quad 0<e^{L}<e^{M}<1, \\
\\
i, j=1,2 .
\end{array}
$$

$\left(\mathrm{H}_{2}\right) k_{i j}(s), h_{i}(s)$, and $l_{i}(s)(i, j=1,2)$ are nonnegative bounded sequences such that

$$
\begin{gathered}
\sum_{s=0}^{\infty} k_{i j}(s)=1, \quad \sum_{s=0}^{\infty} h_{i}(s)=1, \quad \sum_{s=0}^{\infty} l_{i}(s)=1, \\
i, j=1,2 ; \\
\Theta_{i j}=\sum_{s=0}^{\infty} k_{i j}(s) s<+\infty, \quad \Lambda_{i}=\sum_{s=0}^{\infty} h_{i}(s) s<+\infty, \\
\Upsilon_{i}=\sum_{s=0}^{\infty} l_{i}(s) s<+\infty .
\end{gathered}
$$

According to the biological background of system (5), we only consider the solution of system (5) with the following initial conditions:

$$
\begin{gathered}
x_{i}(s)=\Psi_{i}(s) \geq 0, \quad \Psi_{i}(0)>0, \quad \sup _{k \in Z^{-}} \Psi_{i}(k)<+\infty, \\
i=1,2 ; \\
u(s)=\Phi(s) \geq 0, \quad \Phi(0)>0, \\
\sup _{k \in Z^{-}} \Phi(k)<+\infty,
\end{gathered}
$$

where $s=\cdots,-n,-n+1, \ldots,-1,0$. It is easy to prove that the solution of system (5) which satisfies initial conditions (8) is positive.

We mention here that this is the first time such kind of model is proposed and studied, and, as far as system (5) is concerned, whether the single feedback control variable has influence on the persistent property of the system or not is an interesting problem. The aim of this paper is to investigate the dynamic behaviors of the system (5); in particular, we will find out the answer to the above problem.

The organization of this paper is as follows. We introduce some useful lemmas in the next section and then state and prove the main results in Sections 3, 4, and 5, respectively. Three examples together with their numeric simulations are presented to show the feasibility of the main results in Section 6 . We end this paper by a brief discussion.

\section{Lemmas}

Now, let us consider the following difference equation:

$$
y(k+1)=a y(k)+b
$$

where $a, b$ are positive constants.

Lemma 1 (see [9]). Assume that $|a|<1$; for any initial value $y(0)$, there exists a unique solution $y(k)$ of (9), which can be expressed as follows:

$$
y(k)=a^{k}\left(y(0)-y^{*}\right)+y^{*},
$$

where $y^{*}=b /(1-a)$. Thus, for any solution $y(k)$ of the system (10), we have

$$
\lim _{k \rightarrow+\infty} y(k)=y^{*}
$$


Lemma 2 (see [9]). Let $k \in N_{k_{0}}^{+}=\left\{k_{0}, k_{0}+1, \ldots, k_{0}+l, \ldots\right\}$, $r \geq 0$. For any fixed $k, g(k, r)$ is nondecreasing function with respect to $r$, and, for $k \geq k_{0}$, the following inequalities hold:

$$
y(k+1) \leq g(k, y(k)), \quad u(k+1) \geq g(k, u(k)) .
$$

If $y\left(k_{0}\right) \leq u\left(k_{0}\right)$, then $y(k) \leq u(k)$ for all $k \geq k_{0}$.

Lemma 3 (see [2]). Assume that $r(n)>0, x(n)$ satisfies $x(n)>$ 0 , and

$$
x(n+1) \leq x(n) \exp \{r(n)(1-a x(n))\},
$$

for $n \in\left[n_{1}, \infty\right)$, where $a$ is a positive constant. Then

$$
\limsup _{n \rightarrow+\infty}(n) \leq \frac{1}{a r^{M}} \exp \left(r^{M}-1\right) .
$$

Lemma 4 (see [2]). Assume that $r(n)>0, x(n)$ satisfies $x(n)>$ 0 , and

$$
x(n+1) \geq x(n) \exp \{r(n)(1-a x(n))\},
$$

for $n \in\left[n_{1}, \infty\right)$, lim $\sup _{n \rightarrow+\infty} x(n) \leq x^{*}$, and $x\left(n_{1}\right)>0$, where $a$ and $x^{*}$ are positive constants such that $a x^{*}>1$. Then

$$
\liminf _{n \rightarrow+\infty} x(n) \geq \frac{1}{a} \exp \left(r^{M}\left(1-a x^{*}\right)\right)
$$

Lemma 5 (see [2]). Let $x: Z \rightarrow R$ be a nonnegative bounded sequence, and let $H: Z_{+} \rightarrow R$ be a nonnegative sequence such that $\sum_{n=0}^{\infty} H(n)=1$; then

$$
\begin{aligned}
\liminf _{n \rightarrow+\infty} x(n) & \leq \liminf _{n \rightarrow+\infty} \sum_{s=-\infty}^{n} H(n-s) x(s) \\
& \leq \limsup _{n \rightarrow+\infty} \sum_{s=-\infty}^{n} H(n-s) x(s) \\
& \leq \limsup _{n \rightarrow+\infty} x(n) .
\end{aligned}
$$

\section{Permanence}

Concerned with the persistent property of the system (5), we have the following result.

Theorem 6. Assume that

$$
a_{i}^{L}-b_{i j}^{M} M_{j}-d_{i}^{M} B>0, \quad i, j=1,2, i \neq j,
$$

holds; then, for any positive solution $\left(x_{1}(n), x_{2}(n), u(n)\right)$ of the system (5), we have

$$
\begin{gathered}
m_{i} \leq \liminf _{n \rightarrow+\infty} x_{i}(n)<\limsup _{n \rightarrow+\infty} x_{i}(n) \leq M_{i}, \\
A \leq \liminf _{n \rightarrow+\infty} u(n)<\limsup _{n \rightarrow+\infty} u(n) \leq B,
\end{gathered}
$$

where

$$
\begin{aligned}
m_{i} & =\frac{a_{i}^{L}-b_{i j}^{M} M_{j}-d_{i}^{M} B}{b_{i i}^{M}} \exp \left(a_{i}^{L}-b_{i j}^{M} M_{j}-d_{i}^{M} B-b_{i i}^{M} M_{i}\right), \\
M_{i} & =\frac{1}{b_{i i}^{L}} \exp \left(a_{i}^{M}-1\right), \quad i, j=1,2, i \neq j, \\
A & =\frac{c_{1}^{L} m_{1}+c_{2}^{L} m_{2}}{e^{M}}, \quad B=\frac{c_{1}^{M} M_{1}+c_{2}^{M} M_{2}}{e^{L}} .
\end{aligned}
$$

Proof. From the first and second equations of system (5), we have

$$
x_{i}(n+1) \leq x_{i}(n) \exp \left[a_{i}(n)\left(1-\frac{b_{i i}^{L}}{a_{i}^{M}} x_{i}(n)\right)\right] .
$$

And so, from Lemma 3, we can obtain

$$
\limsup _{n \rightarrow+\infty} x_{i}(n) \leq \frac{1}{b_{i i}^{L}} \exp \left(a_{i}^{M}-1\right) \stackrel{\text { def }}{=} M_{i} .
$$

According to Lemma 5, from the above inequality we have

$$
\begin{aligned}
& \limsup _{n \rightarrow+\infty} \sum_{s=0}^{\infty} l_{i}(s) x_{i}(n-s) \\
& \quad=\limsup _{n \rightarrow+\infty} \sum_{s=-\infty}^{n} l_{i}(n-s) x_{i}(s) \\
& \quad \leq \limsup _{n \rightarrow+\infty} x_{i}(n) \leq M_{i} .
\end{aligned}
$$

For any $\varepsilon>0$, there exists a positive integer $n_{1}$ such that

$$
\sum_{s=0}^{\infty} l_{i}(s) x_{i}(n-s) \leq M_{i}+\varepsilon \quad \forall n>n_{1} .
$$

By the third equation of system (5), we have

$$
u(n+1) \leq\left(1-e^{L}\right) u(n)+c_{1}^{M}\left(M_{1}+\varepsilon\right)+c_{2}^{M}\left(M_{2}+\varepsilon\right) .
$$

Hence, by applying Lemmas 1 and 2 to (25), we obtain

$$
\limsup _{n \rightarrow+\infty} u(n) \leq \frac{c_{1}^{M}\left(M_{1}+\varepsilon\right)+c_{2}^{M}\left(M_{2}+\varepsilon\right)}{e^{L}} .
$$

Setting $\varepsilon \rightarrow 0$, it follows that

$$
\limsup _{n \rightarrow+\infty} u(n) \leq \frac{c_{1}^{M} M_{1}+c_{2}^{M} M_{2}}{e^{L}} \stackrel{\text { def }}{=} B .
$$

Condition (18) implies that, for enough small positive constant $\varepsilon_{1}$, the following inequalities hold:

$$
a_{i}^{L}-b_{i j}^{M}\left(M_{j}+\varepsilon_{1}\right)-d_{i}^{M}\left(B+\varepsilon_{1}\right)>0, \quad i, j=1,2, i \neq j .
$$


For the above $\varepsilon_{1}$, it follows from (22) and (27) that there exists a positive integer $n_{2} \geq n_{1}$ such that

$$
\begin{gathered}
\sum_{s=0}^{\infty} h_{i}(s) u(n-s) \leq B+\varepsilon_{1}, \\
\sum_{s=0}^{\infty} k_{i j}(s) x_{i}(n-s) \leq M_{i}+\varepsilon_{1} \quad \forall n>n_{2} .
\end{gathered}
$$

Thus, for all $n>n_{2}$, from (28), (29), and the first two equations of system (5), we have

$$
\begin{aligned}
& x_{i}(n+1) \geq x_{i}(n) \exp \{\left(a_{i}^{L}-b_{i j}^{M}\left(M_{j}+\varepsilon_{1}\right) d_{i}^{M}\left(B+\varepsilon_{1}\right)\right) \\
&\left.\times\left(1-D_{i}^{\varepsilon} x_{i}(n)\right)\right\},
\end{aligned}
$$

where $D_{i}^{\varepsilon}=b_{i i}^{M} /\left(a_{i}^{L}-b_{i j}^{M}\left(M_{j}+\varepsilon_{1}\right)-d_{i}^{M}\left(B+\varepsilon_{1}\right)\right)$ for $i, j=1,2$, $i \neq j$.

Noticing that

$$
\frac{b_{i i}^{M}}{b_{i i}^{L}} \geq 1, \quad \frac{\exp \left(a_{i}^{M}-1\right)}{a_{i}^{L}-b_{i j}^{M}\left(M_{j}+\varepsilon_{1}\right)-d_{i}^{M}\left(B+\varepsilon_{1}\right)}>1,
$$

then

$$
\begin{aligned}
D_{i}^{\varepsilon} \cdot M_{i}= & \frac{b_{i i}^{M}}{a_{i}^{L}-b_{i j}^{M}\left(M_{j}+\varepsilon_{1}\right)-d_{i}^{M}\left(B+\varepsilon_{1}\right)} \\
& \cdot \frac{1}{b_{i i}^{L}} \exp \left(a_{i}^{M}-1\right)>1 .
\end{aligned}
$$

Hence, according to Lemma 4, we have

$$
\begin{aligned}
& \liminf _{n \rightarrow+\infty} x_{i}(n) \\
& \quad \geq \frac{1}{D_{i}^{\varepsilon}} \cdot \exp \left(a_{i}^{L}-b_{i j}^{M}\left(M_{j}+\varepsilon_{1}\right)-d_{i}^{M}\left(B+\varepsilon_{1}\right)-b_{i i}^{M} M_{i}\right) .
\end{aligned}
$$

Setting $\varepsilon_{1} \rightarrow 0$, it follows that

$$
\begin{aligned}
& \liminf _{n \rightarrow+\infty} x_{i}(n) \\
& \quad \geq \frac{1}{D_{i}} \cdot \exp \left(a_{i}^{L}-b_{i j}^{M} M_{j}-d_{i}^{M} B-b_{i i}^{M} M_{i}\right) \stackrel{\text { def }}{=} m_{i},
\end{aligned}
$$

where $D_{i}=b_{i i}^{M} /\left(a_{i}^{L}-b_{i j}^{M} M_{j}-d_{i}^{M} B\right), i=1,2$.

According to Lemma 5, from (34) we have that, for any $\varepsilon_{2}>0$ small enough (without loss of generality, assume that $\left.\varepsilon<(1 / 2) \min _{i}\left\{m_{i}\right\}\right)$, there exists an $n_{3}>n_{2}$, such that

$$
\sum_{s=0}^{\infty} l_{i}(s) x_{i}(n-s) \geq m_{i}-\varepsilon_{2} \quad \forall n \geq n_{3} .
$$

For $n \geq n_{3}$, from (35) and the last equation of system (5), we have

$$
u(n+1) \geq\left(1-e^{M}\right) u(n)+c_{1}^{L}\left(m_{1}-\varepsilon_{2}\right)+c_{2}^{L}\left(m_{2}-\varepsilon_{2}\right) .
$$

Hence, by applying Lemmas 1 and 2 to (36), we obtain

$$
\limsup _{n \rightarrow+\infty} u(n) \geq \frac{c_{1}^{L}\left(m_{1}-\varepsilon_{2}\right)+c_{2}^{L}\left(m_{2}-\varepsilon_{2}\right)}{e^{M}} .
$$

Setting $\varepsilon_{2} \rightarrow 0$, it follows that

$$
\limsup _{n \rightarrow+\infty}(n) \geq \frac{c_{1}^{L} m_{1}+c_{2}^{L} m_{2}}{e^{M}} \stackrel{\text { def }}{=} A .
$$

This ends the proof Theorem 6 .

\section{Global Attractivity}

Concerned with the stability property of the system (5), we have the following result.

Theorem 7. Assume that there exist positive constants $\alpha_{1}, \alpha_{2}$, and $\alpha_{3}$ such that

$$
\begin{gathered}
\alpha_{1} A_{11}-\alpha_{2} b_{21}^{M}-\alpha_{3} c_{1}^{M}>0 \\
\alpha_{2} A_{22}-\alpha_{1} b_{12}^{M}-\alpha_{3} c_{2}^{M}>0 \\
\alpha_{3} e^{L}-\alpha_{1} d_{1}^{M}-\alpha_{2} d_{2}^{M}>0
\end{gathered}
$$

hold; then, for any two positive solutions $\left(x_{1}(n), x_{2}(n), u(n)\right)$ and $\left(x_{1}^{*}(n), x_{2}^{*}(n), u^{*}(n)\right)$ of system $(5)$, we have

$$
\lim _{n \rightarrow+\infty}\left(x_{i}(n)-x_{i}^{*}(n)\right)=0, \quad \lim _{n \rightarrow+\infty}\left(u(n)-u^{*}(n)\right)=0,
$$

where

$$
A_{i i}=\min \left\{b_{i i}^{L}, \frac{2}{M_{i}}-b_{i i}^{M}\right\}, \quad i=1,2
$$

Proof. By (39), we can choose enough small positive constants $\delta$ and $\varepsilon$ such that

$$
\begin{aligned}
& \alpha_{1} A_{11}^{\varepsilon}-\alpha_{2} b_{21}^{M}-\alpha_{3} c_{1}^{M}>\delta, \\
& \alpha_{2} A_{22}^{\varepsilon}-\alpha_{1} b_{12}^{M}-\alpha_{3} c_{2}^{M}>\delta, \\
& \alpha_{3} e^{L}-\alpha_{1} d_{1}^{M}-\alpha_{2} d_{2}^{M}>\delta,
\end{aligned}
$$

where

$$
A_{i i}^{\varepsilon}=\min \left\{b_{i i}^{L}, \frac{2}{M_{i}+\varepsilon}-b_{i i}^{M}\right\}, \quad i=1,2 .
$$

Let $\left(x_{1}(n), x_{2}(n), u(n)\right)$ be any positive solution of system (5). For the above $\varepsilon$, from (22) and (27), there exists an enough large $n^{*}>n_{1}$, such that

$$
x_{i}(n)<M_{i}+\varepsilon, \quad u(n)<B+\varepsilon \quad \forall n \geq n^{*}, i=1,2 .
$$

Now, let us define a Lyapunov functional

$$
V(n)=\alpha_{1} V_{1}(n)+\alpha_{2} V_{2}(n)+\alpha_{3} V_{3}(n),
$$


where $\alpha_{i}, i=1,2,3$, are positive constants:

$$
\begin{aligned}
V_{1}(n)= & \left|\ln x_{1}(n)-\ln x_{1}^{*}(n)\right|+\sum_{s=0}^{\infty} k_{12}(s) \\
& \times \sum_{q=n-s}^{n-1} b_{12}(q+s)\left|x_{2}(q)-x_{2}^{*}(q)\right|+\sum_{s=0}^{\infty} h_{1}(s) \\
& \times \sum_{q=n-s}^{n-1} d_{1}(q+s)\left|u(q)-u^{*}(q)\right| \\
V_{2}(n)= & \left|\ln x_{2}(n)-\ln x_{2}^{*}(n)\right|+\sum_{s=0}^{\infty} k_{21}(s) \\
& \times \sum_{q=n-s}^{n-1} b_{21}(q+s)\left|x_{1}(q)-x_{1}^{*}(q)\right| \\
& +\sum_{s=0}^{\infty} h_{2}(s) \sum_{q=n-s}^{n-1} d_{2}(q+s)\left|u(q)-u^{*}(q)\right| \\
& \times \sum_{q=n-s}^{n-1} c_{i}(q+s)\left|x_{i}(q)-x_{i}^{*}(q)\right| \\
V_{3}(n)= & \left|u(n)-u^{*}(n)\right|+\sum_{i=1}^{2} \sum_{s=0}^{\infty} l_{i}(s) \\
&
\end{aligned}
$$

Then, from the definition of $V_{i}(n), i=1,2,3$, one could easily see that $V(n) \geq 0$ for all $n \in Z^{+}$. Also, for any fixed $n^{*} \in Z^{+}$,

$$
\begin{aligned}
& V\left(n^{*}\right) \\
& =\alpha_{1}\left(\left|\ln x_{1}\left(n^{*}\right)-\ln x_{1}^{*}\left(n^{*}\right)\right|\right. \\
& +\sum_{s=0}^{\infty} k_{12}(s) \sum_{q=n^{*}-s}^{n^{*}-1} b_{12}(q+s)\left|x_{2}(q)-x_{2}^{*}(q)\right| \\
& \left.+\sum_{s=0}^{\infty} h_{1}(s) \sum_{q=n^{*}-s}^{n^{*}-1} d_{1}(q+s)\left|u(q)-u^{*}(q)\right|\right) \\
& +\alpha_{2}\left(\left|\ln x_{2}\left(n^{*}\right)-\ln x_{2}^{*}\left(n^{*}\right)\right|\right. \\
& +\sum_{s=0}^{\infty} k_{21}(s) \sum_{q=n^{*}-s}^{n^{*}-1} b_{21}(q+s)\left|x_{1}(q)-x_{1}^{*}(q)\right| \\
& \left.+\sum_{s=0}^{\infty} h_{2}(s) \sum_{q=n^{*}-s}^{n^{*}-1} d_{2}(q+s)\left|u(q)-u^{*}(q)\right|\right)
\end{aligned}
$$

$$
\begin{aligned}
& +\alpha_{3}\left(\left|u\left(n^{*}\right)-u^{*}\left(n^{*}\right)\right|\right. \\
& \left.+\sum_{i=1}^{2} \sum_{s=0}^{\infty} l_{i}(s) \sum_{q=n^{*}-s}^{n^{*}-1} c_{i}(q+s)\left|x_{i}(q)-x_{i}^{*}(q)\right|\right) \\
& \leq \alpha_{1}\left(\left|\ln x_{1}\left(n^{*}\right)-\ln x_{1}^{*}\left(n^{*}\right)\right|\right. \\
& +b_{12}^{M} \sup _{q \in Z, q \leq n^{*}}\left|x_{2}(q)-x_{2}^{*}(q)\right| \sum_{s=0}^{\infty} k_{12}(s) s \\
& \left.+d_{1}^{M} \sup _{q \in Z, q \leq n^{*}}\left|u(q)-u^{*}(q)\right| \sum_{s=0}^{\infty} h_{1}(s) s\right) \\
& +\alpha_{2}\left(\left|\ln x_{2}\left(n^{*}\right)-\ln x_{2}^{*}\left(n^{*}\right)\right|\right. \\
& +b_{21}^{M} \sup _{q \in Z, q \leq n^{*}}\left|x_{1}(q)-x_{1}^{*}(q)\right| \\
& \times \sum_{s=0}^{\infty} k_{21}(s) s+d_{2}^{M} \sup _{q \in Z, q \leq n^{*}}\left|u(q)-u^{*}(q)\right| \\
& \left.\times \sum_{s=0}^{\infty} h_{2}(s) s\right) \\
& +\alpha_{3}\left(\left|u\left(n^{*}\right)-u^{*}\left(n^{*}\right)\right|\right. \\
& +c_{i}^{M} \sup _{q \in Z, q \leq n^{*}}\left|x_{i}(q)-x_{i}^{*}(q)\right| \\
& \left.\times \sum_{i=1}^{2} \sum_{s=0}^{\infty} l_{i}(s) s\right)<+\infty .
\end{aligned}
$$

Also, from the first equation of system (5) and using the Mean Value Theorem, we can obtain

$$
\begin{aligned}
\Delta V_{1}(n) \leq & \left|\ln x_{1}(n)-\ln x_{1}^{*}(n)-b_{11}(n)\left(x_{1}(n)-x_{1}^{*}(n)\right)\right| \\
& -\left|\ln x_{1}(n)-\ln x_{1}^{*}(n)\right|+b_{12}(n) \\
& \times \sum_{s=0}^{\infty} k_{12}(s)\left|x_{2}(n-s)-x_{2}^{*}(n-s)\right| \\
& +d_{1}(n) \sum_{s=0}^{\infty} h_{1}(s)\left|u(n-s)-u^{*}(n-s)\right| \\
& +\sum_{s=0}^{\infty} k_{12}(s)\left(b_{12}(n+s)\left|x_{2}(n)-x_{2}^{*}(n)\right|\right. \\
& \left.\quad-b_{12}(n)\left|x_{2}(n-s)-x_{2}^{*}(n-s)\right|\right)
\end{aligned}
$$




$$
\begin{aligned}
& +\sum_{s=0}^{\infty} h_{1}(s)\left(d_{1}(n+s)\left|u(n)-u^{*}(n)\right|\right. \\
& \left.-d_{1}(n)\left|u(n-s)-u^{*}(n-s)\right|\right) \\
\leq & -\left(\frac{1}{\varphi_{1}(n)}-\left|\frac{1}{\varphi_{1}(n)}-b_{11}(n)\right|\right)\left|x_{1}(n)-x_{1}^{*}(n)\right| \\
& +\sum_{s=0}^{\infty} k_{12}(s) b_{12}(n+s)\left|x_{2}(n)-x_{2}^{*}(n)\right| \\
& +\sum_{s=0}^{\infty} h_{1}(s) d_{1}(n+s)\left|u(n)-u^{*}(n)\right| \\
\leq & -\left(\frac{1}{\varphi_{1}(n)}-\left|\frac{1}{\varphi_{1}(n)}-b_{11}(n)\right|\right)\left|x_{1}(n)-x_{1}^{*}(n)\right| \\
& +b_{12}^{M}\left|x_{2}(n)-x_{2}^{*}(n)\right|+d_{1}^{M}\left|u(n)-u^{*}(n)\right|,
\end{aligned}
$$

where $\varphi_{1}(n)$ lies between $x_{1}(n)$ and $x_{1}^{*}(n)$.

Similarly to the analysis of (48), we can obtain

$$
\begin{aligned}
\Delta V_{2}(n) & \\
\leq & -\left(\frac{1}{\varphi_{2}(n)}-\left|\frac{1}{\varphi_{2}(n)}-b_{22}(n)\right|\right)\left|x_{2}(n)-x_{2}^{*}(n)\right| \\
& +b_{21}^{M}\left|x_{1}(n)-x_{1}^{*}(n)\right|+d_{2}^{M}\left|u(n)-u^{*}(n)\right|
\end{aligned}
$$

where $\varphi_{2}(n)$ lies between $x_{2}(n)$ and $x_{2}^{*}(n)$ :

$$
\begin{aligned}
\Delta V_{3}(n)= & V_{3}(n+1)-V_{3}(n) \\
\leq & -e(n)\left|u(n)-u^{*}(n)\right|+\sum_{i=1}^{2} c_{i}(n) \\
& \times \sum_{s=0}^{\infty} l_{i}(s)\left|x_{i}(n-s)-x_{i}^{*}(n-s)\right| \\
& +\sum_{i=1}^{2} \sum_{s=0}^{\infty} l_{i}(s)\left(c_{i}(n+s)\right. \\
\quad \times\left|x_{i}(n)-x_{i}^{*}(n)\right|-c_{i}(n) & \left.\times\left|x_{i}(n-s)-x_{i}^{*}(n-s)\right|\right) \\
= & -e(n)\left|u(n)-u^{*}(n)\right| \\
& +\sum_{i=1}^{2} \sum_{s=0}^{\infty} l_{i}(s) c_{i}(n+s)\left|x_{i}(n)-x_{i}^{*}(n)\right| \\
\leq & -e^{L}\left|u(n)-u^{*}(n)\right| \\
& +\sum_{i=1}^{2} c_{i}^{M}\left|x_{i}(n)-x_{i}^{*}(n)\right| .
\end{aligned}
$$

From (42)-(44) and (48)-(50), for any $n \geq n^{*}$, we have

$$
\begin{aligned}
& \Delta V(n) \leq \alpha_{1}\left\{-\left(\frac{1}{\varphi_{1}(n)}-\left|\frac{1}{\varphi_{1}(n)}-b_{11}(n)\right|\right)\right. \\
& \times\left|x_{1}(n)-x_{1}^{*}(n)\right| \\
& +b_{12}^{M}\left|x_{2}(n)-x_{2}^{*}(n)\right| \\
& \left.+d_{1}^{M}\left|u(n)-u^{*}(n)\right|\right\} \\
& +\alpha_{2}\left\{-\left(\frac{1}{\varphi_{2}(n)}-\left|\frac{1}{\varphi_{2}(n)}-b_{22}(n)\right|\right)\right. \\
& \times\left|x_{2}(n)-x_{2}^{*}(n)\right| \\
& +b_{21}^{M}\left|x_{1}(n)-x_{1}^{*}(n)\right| \\
& \left.+d_{2}^{M}\left|u(n)-u^{*}(n)\right|\right\} \\
& +\alpha_{3}\left\{-e^{L}\left|u(n)-u^{*}(n)\right|\right. \\
& \left.+\sum_{i=1}^{2} c_{i}^{M}\left|x_{i}(n)-x_{i}^{*}(n)\right|\right\} \\
& \leq-\left\{\alpha_{1} \min \left[b_{11}^{L}, \frac{2}{M_{1}+\varepsilon}-b_{11}^{M}\right]-\alpha_{2} b_{21}^{M}-\alpha_{3} c_{1}^{M}\right\} \\
& \times\left|x_{1}(n)-x_{1}^{*}(n)\right| \\
& -\left\{\alpha_{2} \min \left[b_{22}^{L}, \frac{2}{M_{2}+\varepsilon}-b_{22}^{M}\right]-\alpha_{1} b_{12}^{M}-\alpha_{3} c_{2}^{M}\right\} \\
& \times\left|x_{2}(n)-x_{2}^{*}(n)\right| \\
& -\left\{\alpha_{3} e^{L}-\alpha_{1} d_{1}^{M}-\alpha_{2} d_{2}^{M}\right\}\left|u(n)-u^{*}(n)\right| \\
& =-\left\{\alpha_{1} A_{11}^{\varepsilon}-\alpha_{2} b_{21}^{M}-\alpha_{3} c_{1}^{M}\right\}\left|x_{1}(n)-x_{1}^{*}(n)\right| \\
& -\left\{\alpha_{2} A_{22}^{\varepsilon}-\alpha_{1} b_{12}^{M}-\alpha_{3} c_{2}^{M}\right\}\left|x_{2}(n)-x_{2}^{*}(n)\right| \\
& -\left\{\alpha_{3} e^{L}-\alpha_{1} d_{1}^{M}-\alpha_{2} d_{2}^{M}\right\}\left|u(n)-u^{*}(n)\right| \\
& \leq-\delta\left(\sum_{i=1}^{2}\left|x_{i}(n)-x_{i}^{*}(n)\right|+\left|u(n)-u^{*}(n)\right|\right) \text {. }
\end{aligned}
$$

Summating both sides of the above inequalities from $n^{*}$ to $n$, we have

$$
\begin{aligned}
& \sum_{p=n^{*}}^{n}(V(p+1)-V(p)) \\
& \quad \leq-\delta \sum_{p=n^{*}}^{n}\left(\sum_{i=1}^{2}\left|x_{i}(p)-x_{i}^{*}(p)\right|+\left|u(p)-u^{*}(p)\right|\right) .
\end{aligned}
$$


Hence

$$
\begin{aligned}
& V(n+1)+\delta \sum_{p=n^{*}}^{n}\left(\sum_{i=1}^{2}\left|x_{i}(p)-x_{i}^{*}(p)\right|+\left|u(p)-u^{*}(p)\right|\right) \\
& \quad \leq V\left(n^{*}\right)<+\infty .
\end{aligned}
$$

Then, we have

$$
\sum_{p=n^{*}}^{n}\left(\sum_{i=1}^{2}\left|x_{i}(p)-x_{i}^{*}(p)\right|+\left|u(p)-u^{*}(p)\right|\right) \leq \frac{V\left(n^{*}\right)}{\delta} .
$$

Therefore

$$
\sum_{p=n^{*}}^{\infty}\left(\sum_{i=1}^{2}\left|x_{i}(p)-x_{i}^{*}(p)\right|+\left|u(p)-u^{*}(p)\right|\right)<+\infty,
$$

which means that

$$
\lim _{n \rightarrow+\infty}\left(\sum_{i=1}^{2}\left|x_{i}(n)-x_{i}^{*}(n)\right|+\left|u(n)-u^{*}(n)\right|\right)=0 .
$$

Consequently

$$
\begin{gathered}
\lim _{n \rightarrow+\infty}\left(x_{i}(n)-x_{i}^{*}(n)\right)=0, \quad i=1,2, \\
\lim _{n \rightarrow+\infty}\left(u(n)-u^{*}(n)\right)=0 .
\end{gathered}
$$

This completes the proof of Theorem 7

\section{Extinction}

Concerned with the extinction property of the system (5), when the coefficients of the third equation are all constants, we could establish the following results.

Theorem 8. Assume that

$$
\frac{a_{1}^{M}}{a_{2}^{L}}<\frac{b_{11}^{L} e+d_{1}^{L} c_{1}}{b_{21}^{M} e+d_{2}^{M} c_{1}}, \quad \frac{a_{1}^{M}}{a_{2}^{L}}<\frac{b_{12}^{L} e+d_{1}^{L} c_{2}}{b_{22}^{M} e+d_{2}^{M} c_{2}}
$$

hold; let $\left(x_{1}(n), x_{2}(n), u(n)\right)$ be any positive solution of system (5); then

$$
\lim _{n \rightarrow+\infty} x_{1}(n)=0
$$

Theorem 9. Assume that

$$
\frac{a_{1}^{L}}{a_{2}^{M}}>\frac{b_{11}^{M} e+d_{1}^{M} c_{1}}{b_{21}^{L} e+d_{2}^{L} c_{1}}, \quad \frac{a_{1}^{L}}{a_{2}^{M}}>\frac{b_{12}^{M} e+d_{1}^{M} c_{2}}{b_{22}^{L} e+d_{2}^{L} c_{2}}
$$

hold; let $\left(x_{1}(n), x_{2}(n), u(n)\right)$ be any positive solution of system (5); then

$$
\lim _{n \rightarrow+\infty} x_{2}(n)=0
$$

Proof of Theorem 8. By conditions (58), we can choose positive constants $\alpha_{n 1}, \alpha_{n 2}$ such that

$$
\begin{aligned}
& \frac{a_{1}^{M}}{a_{2}^{L}}<\frac{\alpha_{n 2}}{\alpha_{n 1}}<\frac{b_{11}^{L} e+d_{1}^{L} c_{1}}{b_{21}^{M} e+d_{2}^{M} c_{1}}, \\
& \frac{a_{1}^{M}}{a_{2}^{L}}<\frac{\alpha_{n 2}}{\alpha_{n 1}}<\frac{b_{12}^{L} e+d_{1}^{L} c_{2}}{b_{22}^{M} e+d_{2}^{M} c_{2}} .
\end{aligned}
$$

Thus, there exists a positive constant $\eta$ such that

$$
\alpha_{n 1} a_{1}^{M}-\alpha_{n 2} a_{2}^{L}<-\eta<0
$$

$$
\begin{aligned}
& \alpha_{n 2} b_{21}^{M}-\alpha_{n 1} b_{11}^{L}+\frac{\left(\alpha_{n 2} d_{2}^{M}-\alpha_{n 1} d_{1}^{L}\right) c_{1}}{e}<0, \\
& \alpha_{n 2} b_{22}^{M}-\alpha_{n 1} b_{12}^{L}+\frac{\left(\alpha_{n 2} d_{2}^{M}-\alpha_{n 1} d_{1}^{L}\right) c_{2}}{e}<0 .
\end{aligned}
$$

There exists a constant $\alpha_{n 3}$ such that

$$
\begin{gathered}
\frac{\alpha_{n 2} d_{2}^{M}-\alpha_{n 1} d_{1}^{L}}{e} \\
<\alpha_{n 3} \Longrightarrow \alpha_{n 2} d_{2}^{M}-\alpha_{n 1} d_{1}^{L}-\alpha_{n 3} e<0, \\
\alpha_{n 2} b_{21}^{M}-\alpha_{n 1} b_{11}^{L}+\alpha_{n 3} c_{1}<0, \\
\alpha_{n 2} b_{22}^{M}-\alpha_{n 1} b_{12}^{L}+\alpha_{n 3} c_{2}<0 .
\end{gathered}
$$

Consider the following discrete Lyapunov functional:

$$
\begin{aligned}
V_{4}(n)=x_{1}^{\alpha_{n 1}}(n) & x_{2}^{-\alpha_{n 2}}(n) \\
& \times \exp \left\{\alpha_{n 3} u(n)-\alpha_{n 1} \sum_{s=0}^{\infty} k_{12}(s)\right. \\
& \times \sum_{q=n-s}^{n-1} b_{12}(q+s) x_{2}(q) \\
& -\alpha_{n 1} \sum_{s=0}^{\infty} h_{1}(s) \sum_{q=n-s}^{n-1} d_{1}(q+s) u(q) \\
& +\alpha_{n 2} \sum_{s=0}^{\infty} k_{21}(s) \sum_{q=n-s}^{n-1} b_{21}(q+s) x_{1}(q) \\
& +\alpha_{n 2} \sum_{s=0}^{\infty} h_{2}(s) \sum_{q=n-s}^{n-1} d_{2}(q+s) u(q) \\
& \left.+\alpha_{n 3} \sum_{i=1}^{2} \sum_{s=0}^{\infty} l_{i}(s) \sum_{q=n-s}^{n-1} c_{i} x_{i}(q)\right\}
\end{aligned}
$$


From (65), we obtain

$$
\begin{aligned}
& \frac{V_{4}(n+1)}{V_{4}(n)} \\
& =\exp \left\{\alpha _ { n 1 } \left(a_{1}(n)-b_{11}(n) x_{1}(n)\right.\right. \\
& -b_{12}(n) \sum_{s=0}^{\infty} k_{12}(s) x_{2}(n-s) \\
& \left.-d_{1}(n) \sum_{s=0}^{\infty} h_{1}(s) u(n-s)\right) \\
& -\alpha_{n 2}\left(a_{2}(n)-b_{22}(n) x_{2}(n)\right. \\
& -b_{21}(n) \sum_{s=0}^{\infty} k_{21}(s) x_{1}(n-s) \\
& \left.-d_{2}(n) \sum_{s=0}^{\infty} h_{2}(s) u(n-s)\right) \\
& +\alpha_{n 3}\left(-e u(n)+c_{1} \sum_{s=0}^{\infty} l_{1}(s) x_{1}(n-s)\right. \\
& \left.+c_{2} \sum_{s=0}^{\infty} l_{2}(s) x_{2}(n-s)\right) \\
& -\alpha_{n 1} \sum_{s=0}^{\infty} k_{12}(s)\left(b_{12}(n+s) x_{2}(n)\right. \\
& \left.-b_{12}(n) x_{2}(n-s)\right) \\
& -\alpha_{n 1} \sum_{s=0}^{\infty} h_{1}(s)\left(d_{1}(n+s) u(n)\right. \\
& \left.-d_{1}(n) u(n-s)\right) \\
& +\alpha_{n 2} \sum_{s=0}^{\infty} k_{21}(s)\left(b_{21}(n+s) x_{1}(n)\right. \\
& \left.-b_{21}(n) x_{1}(n-s)\right) \\
& +\alpha_{n 2} \sum_{s=0}^{\infty} h_{2}(s)\left(d_{2}(n+s) u(n)\right. \\
& \left.-d_{2}(n) u(n-s)\right) \\
& +\alpha_{n 3} \sum_{i=1}^{2} \sum_{s=0}^{\infty} l_{i}(s) \\
& \left.\times\left(c_{i} x_{i}(n)-c_{i} x_{i}(n-s)\right)\right\} \\
& \leq \exp \left\{\left(\alpha_{n 1} a_{1}^{M}-\alpha_{n 2} a_{2}^{L}\right)\right.
\end{aligned}
$$

$$
\begin{aligned}
& +\left(\alpha_{n 2} b_{21}^{M}-\alpha_{n 1} b_{11}^{L}+\alpha_{n 3} c_{1}\right) x_{1}(n) \\
& +\left(\alpha_{n 2} b_{22}^{M}-\alpha_{n 1} b_{12}^{L}+\alpha_{n 3} c_{2}\right) x_{2}(n) \\
& \left.+\left(\alpha_{n 2} d_{2}^{M}-\alpha_{n 1} d_{1}^{L}-\alpha_{n 3} e\right) u(n)\right\} .
\end{aligned}
$$

From inequalities (63) and (64), we can obtain

$$
V_{4}(n+1) \leq V_{4}(n) \exp (-\eta)
$$

Therefore

$$
V_{4}(n) \leq V_{4}(0) \exp (-n \eta)
$$

From (22) and (27) we know that there exists an $M>0$ such that

$$
x_{i}(k)<M, \quad u(k)<M \quad \forall k \in Z,
$$

and so

$$
\begin{aligned}
& V_{4}(0) \\
& =x_{1}^{\alpha_{n 1}}(0) x_{2}^{-\alpha_{n 2}}(0) \\
& \times \exp \left\{\alpha_{n 3} u(0)-\alpha_{n 1} \sum_{s=0}^{\infty} k_{12}(s)\right. \\
& \times \sum_{q=-s}^{-1} b_{12}(q+s) x_{2}(q) \\
& -\alpha_{n 1} \sum_{s=0}^{\infty} h_{1}(s) \sum_{q=-s}^{-1} d_{1}(q+s) u(q) \\
& +\alpha_{n 2} \sum_{s=0}^{\infty} k_{21}(s) \sum_{q=-s}^{-1} b_{21}(q+s) x_{1}(q) \\
& +\alpha_{n 2} \sum_{s=0}^{\infty} h_{2}(s) \sum_{q=n-s}^{n-1} d_{2}(q+s) u(q) \\
& \left.+\alpha_{n 3} \sum_{i=1}^{2} \sum_{s=0}^{\infty} l_{i}(s) \sum_{q=-s}^{-1} c_{i} x_{i}(q)\right\} \\
& <x_{1}^{\alpha_{n 1}}(0) x_{2}^{-\alpha_{n 2}}(0) \\
& \times \exp \left\{\left|\alpha_{n 3}\right| M+\alpha_{n 2} M b_{21}^{M} \sum_{s=0}^{\infty} k_{21}(s) s\right. \\
& +\alpha_{n 2} M d_{2}^{M} \sum_{s=0}^{\infty} h_{2}(s) s \\
& \left.+\alpha_{n 3} M c_{i} \sum_{i=1}^{2} \sum_{s=0}^{\infty} l_{i}(s) s\right\}
\end{aligned}
$$




$$
\begin{aligned}
& =x_{1}^{\alpha_{n 1}}(0) x_{2}^{-\alpha_{n 2}}(0) \\
& \times \exp \left\{M \left(\left|\alpha_{n 3}\right|+\alpha_{n 2} b_{21}^{M} \Theta_{21}+\alpha_{n 2} d_{2}^{M} \Lambda_{2}\right.\right. \\
& \left.\left.+\alpha_{n 3} \sum_{i=1}^{2} c_{i} \Upsilon_{i}\right)\right\}<+\infty
\end{aligned}
$$

On the other hand, we also have

$$
\begin{aligned}
& V_{4}(n) \\
& \geq x_{1}^{\alpha_{n 1}}(n) x_{2}^{-\alpha_{n 2}}(n) \\
& \times \exp \left\{-\alpha_{n 1} \sum_{s=0}^{\infty} k_{12}(s) \sum_{q=n-s}^{n-1} b_{12}(q+s) x_{2}(q)\right. \\
& \left.-\alpha_{n 1} \sum_{s=0}^{\infty} h_{1}(s) \sum_{q=n-s}^{n-1} d_{1}(q+s) u(q)\right\} \\
& \geq x_{1}^{\alpha_{n 1}}(n) M^{-\alpha_{n 2}} \\
& \times \exp \left\{-\alpha_{n 1} M b_{12}^{M} \sum_{s=0}^{\infty} k_{12}(s) s\right. \\
& \left.-\alpha_{n 1} M d_{1}^{M} \sum_{s=0}^{\infty} h_{1}(s) s\right\} \\
& =x_{1}^{\alpha_{n 1}}(n) M^{-\alpha_{n 2}} \\
& \times \exp \left\{-\alpha_{n 1} M\left(b_{12}^{M} \Theta_{12}+d_{1}^{M} \Lambda_{1}\right)\right\} .
\end{aligned}
$$

Combining inequalities (68), (70), and (71), we have

$$
x_{1}(n) \leq \lambda \exp \left\{-\frac{\eta}{\alpha_{n 1}} n\right\}
$$

where

$$
\begin{aligned}
\lambda & =\left(V_{4}(0)\right)^{1 / \alpha_{n 1}} M^{\alpha_{n 2} / \alpha_{n 1}} \exp \left\{M\left(b_{12}^{M} \Theta_{12}+d_{1}^{M} \Lambda_{1}\right)\right\} \\
& <+\infty
\end{aligned}
$$

Hence we obtain that

$$
\lim _{n \rightarrow+\infty} x_{1}(n)=0
$$

This ends the proof of Theorem 8.
Proof of Theorem 9. By (60), we can choose positive constants $\beta_{n 1}, \beta_{n 2}$, and $\beta_{n 3}$ and constant $\gamma$ such that

$$
\begin{aligned}
& \frac{a_{1}^{M}}{a_{2}^{L}}>\frac{\beta_{n 2}}{\beta_{n 1}}>\frac{b_{11}^{M} e+d_{1}^{M} c_{1}}{b_{21}^{L} e+d_{2}^{L} c_{1}}, \\
& \frac{a_{1}^{M}}{a_{2}^{L}}>\frac{\beta_{n 2}}{\beta_{n 1}}>\frac{b_{12}^{M} e+d_{1}^{M} c_{2}}{b_{22}^{L} e+d_{2}^{L} c_{2}} . \\
& \beta_{n 2} a_{2}^{M}-\beta_{n 1} a_{1}^{L}<-\gamma<0, \\
& \beta_{n 1} b_{11}^{M}-\beta_{n 2} b_{21}^{L}+\beta_{n 3} c_{1}<0, \\
& \beta_{n 1} b_{12}^{M}-\beta_{n 2} b_{22}^{L}+\beta_{n 3} c_{2}<0, \\
& \beta_{n 1} d_{1}^{M}-\beta_{n 2} d_{2}^{L}-\beta_{n 3} e<0 .
\end{aligned}
$$

Define the following Lyapunov functional:

$$
\begin{aligned}
& V_{5}(n) x_{1}^{-\beta_{n 1}}(n) x_{2}^{\beta_{n 2}}(n) \\
& \times \exp \left\{\beta_{n 3} u(n)+\beta_{n 1} \sum_{s=0}^{\infty} k_{12}(s)\right. \\
& \\
& \times \sum_{q=n-s}^{n-1} b_{12}(q+s) x_{2}(q) \\
&+\beta_{n 1} \sum_{s=0}^{\infty} h_{1}(s) \sum_{q=n-s}^{n-1} d_{1}(q+s) u(q) \\
&-\beta_{n 2} \sum_{s=0}^{\infty} k_{21}(s) \sum_{q=n-s}^{n-1} b_{21}(q+s) x_{1}(q) \\
& \quad-\beta_{n 2} \sum_{s=0}^{\infty} h_{2}(s) \sum_{q=n-s}^{n-1} d_{2}(q+s) u(q) \\
&\left.+\beta_{n 3} \sum_{i=1}^{2} \sum_{s=0}^{\infty} l_{i}(s) \sum_{q=n-s}^{n-1} c_{i} x_{i}(q)\right\} .
\end{aligned}
$$

From (76), we have

$$
\begin{aligned}
& \frac{V_{5}(n+1)}{V_{5}(n)} \\
& \leq \exp \left\{\left(\beta_{n 2} a_{2}^{M}-\beta_{n 1} a_{1}^{L}\right)\right. \\
& +\left(\beta_{n 1} b_{11}^{M}-\beta_{n 2} b_{21}^{L}+\beta_{n 3} c_{1}\right) x_{1}(n) \\
& +\left(\beta_{n 1} b_{12}^{M}-\beta_{n 2} b_{22}^{L}+\beta_{n 3} c_{2}\right) x_{2}(n) \\
& \left.+\left(\beta_{n 1} d_{1}^{M}-\beta_{n 2} d_{2}^{L}-\beta_{n 3} e\right) u(n)\right\} \\
& \leq \exp \{-\gamma\} .
\end{aligned}
$$

Similarly to the analysis of (68)-(73), we have

$$
\lim _{n \rightarrow+\infty} x_{2}(n)=0 \text {. }
$$

This ends the proof of Theorem 9. 
From Theorems 8 and 9 we know that, under some suitable assumption, one of the species in the system may be driven to extinction; in this case, one interesting problem is to investigate the stability property of the rest of the species.

Consider the following discrete equations:

$$
\begin{gathered}
x_{2}(n+1)=x_{2}(n) \exp \left\{a_{2}(n)-b_{22}(n) x_{2}(n)\right. \\
\left.-d_{2}(n) \sum_{s=0}^{\infty} h_{2}(s) u(n-s)\right\}, \\
\begin{aligned}
u(n+1)=u(n)(1-e)+c_{2} \sum_{s=0}^{\infty} l_{2}(s) x_{2}(n-s) . \\
x_{1}(n+1)=x_{1}(n) \exp \left\{a_{1}(n)-b_{11}(n) x_{1}(n)\right.
\end{aligned} \\
\left.-d_{1}(n) \sum_{s=0}^{\infty} h_{1}(s) u(n-s)\right\} \\
\begin{array}{c}
u(n+1)=u(n)(1-e)+c_{1} \sum_{s=0}^{\infty} l_{1}(s) x_{1}(n-s) .
\end{array}
\end{gathered}
$$

Theorem 10. Assume that (58) holds and also

$$
d_{2}^{M}<\frac{A_{22} e}{c_{2}}
$$

holds; then, for any positive solution $\left(x_{1}(n), x_{2}(n), u(n)\right)$ of system (5) and any positive solution $\left(x_{2}^{*}(n), u^{*}(n)\right)$ of system (79), we have

$$
\begin{gathered}
\lim _{n \rightarrow+\infty} x_{1}(n)=0, \quad \lim _{n \rightarrow+\infty}\left(x_{2}(n)-x_{2}^{*}(n)\right)=0, \\
\lim _{n \rightarrow+\infty}\left(u(n)-u^{*}(n)\right)=0,
\end{gathered}
$$

where $A_{22}$ is defined in Theorem 7.

Theorem 11. Assume that (60) holds and also

$$
d_{1}^{M}<\frac{A_{11} e}{c_{1}}
$$

holds; then, for any positive solution $\left(x_{1}(n), x_{2}(n), u(n)\right)$ of system (5) and any positive solution $\left(x_{1}^{*}(n), u^{*}(n)\right)$ of system (80), we have

$$
\begin{gathered}
\lim _{n \rightarrow+\infty}\left(x_{1}(n)-x_{1}^{*}(n)\right)=0, \quad \lim _{n \rightarrow+\infty} x_{2}(n)=0, \\
\lim _{n \rightarrow+\infty}\left(u(n)-u^{*}(n)\right)=0,
\end{gathered}
$$

Proof of Theorem 10. By conditions (81), we can choose positive constants $\beta_{1}, \beta_{2}$ such that

$$
\frac{e}{d_{2}^{M}}>\frac{\beta_{1}}{\beta_{2}}>\frac{c_{2}}{A_{22}} .
$$

Thus, there exist enough small positive constants $\delta$ and $\varepsilon$ such that

$$
\begin{aligned}
& \beta_{1} A_{22}^{\varepsilon}-\beta_{2} c_{2}>\delta \\
& \beta_{2} e-\beta_{1} d_{2}^{M}>\delta
\end{aligned}
$$

where $A_{22}^{\varepsilon}$ is defined in(43)

Now, we define a Lyapunov functional

$$
Q_{1}(n)=\beta_{1} G_{1}(n)+\beta_{2} H_{1}(n),
$$

where

$$
\begin{aligned}
G_{1}(n)= & \left|\ln x_{2}(n)-\ln x_{2}^{*}(n)\right|+\sum_{s=0}^{\infty} k_{21}(s) \\
& \times \sum_{q=n-s}^{n-1} b_{21}(q+s) x_{1}(q)+\sum_{s=0}^{\infty} h_{2}(s) \\
& \times \sum_{q=n-s}^{n-1} d_{2}(q+s)\left|u(q)-u^{*}(q)\right| ; \\
H_{1}(n)= & \left|u(n)-u^{*}(n)\right|+\sum_{s=0}^{\infty} l_{1}(s) \\
& \times \sum_{q=n-s}^{n-1} c_{1} x_{1}(q)+\sum_{s=0}^{\infty} l_{2}(s) \\
& \times \sum_{q=n-s}^{n-1} c_{2}\left|x_{2}(q)-x_{2}^{*}(q)\right| .
\end{aligned}
$$

Then, from the definition of $G_{1}(n), H_{1}(n)$, one could easily see that $Q_{1}(n) \geq 0$ for all $n \in Z^{+}$. Also, for any fixed $n^{*} \in Z^{+}$, from (69) one could see that

$$
\begin{aligned}
Q_{1}\left(n^{*}\right)= & \beta_{1}\left|\ln x_{2}\left(n^{*}\right)-\ln x_{2}^{*}\left(n^{*}\right)\right| \\
& +\beta_{1} M b_{21}^{M} \sum_{s=0}^{\infty} k_{21}(s) s \\
& +\beta_{1} d_{2}^{M} \sup _{q \in Z^{+}, q \leq n^{*}}\left|u(q)-u^{*}(q)\right| \sum_{s=0}^{\infty} h_{2}(s) s \\
& +\beta_{2}\left|u\left(n^{*}\right)-u^{*}\left(n^{*}\right)\right|+\beta_{2} M c_{1} \sum_{s=0}^{\infty} l_{1}(s) s \\
& +\beta_{2} c_{2} \sup _{q \in Z^{+}, q \leq n^{*}}\left|x_{2}(q)-x_{2}^{*}(q)\right| \sum_{s=0}^{\infty} l_{2}(s) s
\end{aligned}
$$


It follows from the second equation of system (5) and the Mean Value Theorem that

$$
\begin{aligned}
& \Delta G_{1}(n) \\
& \leq\left|\ln x_{2}(n)-\ln x_{2}^{*}(n)-b_{22}(n)\left(x_{2}(n)-x_{2}^{*}(n)\right)\right| \\
& -\left|\ln x_{2}(n)-\ln x_{2}^{*}(n)\right| \\
& +b_{21}(n) \sum_{s=0}^{\infty} k_{21}(s) x_{1}(n-s)+d_{2}(n) \\
& \times \sum_{s=0}^{\infty} h_{2}(s)\left|u(n-s)-u^{*}(n-s)\right| \\
& +\sum_{s=0}^{\infty} k_{21}(s)\left(b_{21}(n+s) x_{1}(n)\right. \\
& \left.-b_{21}(n) x_{1}(n-s)\right) \\
& +\sum_{s=0}^{\infty} h_{2}(s)\left(d_{2}(n+s)\left|u(n)-u^{*}(n)\right|\right. \\
& \left.-d_{2}(n)\left|u(n-s)-u^{*}(n-s)\right|\right) \\
& \leq-\left(\frac{1}{\varphi_{2}(n)}-\left|\frac{1}{\varphi_{2}(n)}-b_{22}(n)\right|\right)\left|x_{2}(n)-x_{2}^{*}(n)\right| \\
& +\sum_{s=0}^{\infty} k_{21}(s) b_{21}(n+s) x_{1}(n) \\
& +\sum_{s=0}^{\infty} h_{2}(s) d_{2}(n+s)\left|u(n)-u^{*}(n)\right| \\
& \leq-\left(\frac{1}{\varphi_{2}(n)}-\left|\frac{1}{\varphi_{2}(n)}-b_{22}(n)\right|\right)\left|x_{2}(n)-x_{2}^{*}(n)\right| \\
& +b_{12}^{M} x_{1}(n)+d_{2}^{M}\left|u(n)-u^{*}(n)\right| \text {. }
\end{aligned}
$$

Also, from the third equation of system (5) we have

$$
\begin{aligned}
& \Delta H_{1}(n)= H_{1}(n+1)-H_{1}(n) \\
& \leq-e\left|u(n)-u^{*}(n)\right| \\
&+c_{1} \sum_{s=0}^{\infty} l_{1}(s) x_{1}(n-s) \\
&+c_{2} \sum_{s=0}^{\infty} l_{2}(s)\left|x_{2}(n-s)-x_{2}^{*}(n-s)\right| \\
&+\sum_{s=0}^{\infty} l_{1}(s)\left(c_{1} x_{1}(n)-c_{1} x_{1}(n-s)\right) \\
&+\sum_{s=0}^{\infty} l_{2}(s)\left(c_{2}\left|x_{2}(n)-x_{2}^{*}(n)\right|\right. \\
&\left.\quad-c_{2}\left|x_{2}(n-s)-x_{2}^{*}(n-s)\right|\right)
\end{aligned}
$$

$$
\begin{aligned}
= & -e\left|u(n)-u^{*}(n)\right|+\sum_{s=0}^{\infty} l_{1}(s) c_{1} x_{1}(n) \\
& +\sum_{s=0}^{\infty} l_{2}(s) c_{2}\left|x_{2}(n)-x_{2}^{*}(n)\right| \\
\leq & -e\left|u(n)-u^{*}(n)\right|+c_{1} x_{1}(n) \\
& +c_{2}\left|x_{2}(n)-x_{2}^{*}(n)\right| .
\end{aligned}
$$

From (70), we have

$$
\sum_{p=n^{*}}^{\infty} x_{1}(p) \leq \sum_{p=n^{*}}^{\infty} \lambda \exp \left\{-\frac{\eta}{\alpha_{n 1}} p\right\}<+\infty .
$$

Therefore, from (44), (88), and (90), for all $n>n^{*}$, we have

$$
\begin{aligned}
\Delta Q_{1}(n) & \beta_{1}\left\{-\left(\frac{1}{\varphi_{2}(n)}-\left|\frac{1}{\varphi_{2}(n)}-b_{22}(n)\right|\right)\left|x_{2}(n)-x_{2}^{*}(n)\right|\right. \\
& \left.+b_{21}^{M} x_{1}(n)+d_{2}^{M}\left|u(n)-u^{*}(n)\right|\right\} \\
& +\beta_{2}\left\{-e\left|u(n)-u^{*}(n)\right|+c_{1} x_{1}(n)+c_{2}\left|x_{2}(n)-x_{2}^{*}(n)\right|\right\} \\
\leq & -\left\{\beta_{1} \min \left[b_{22}^{L}, \frac{2}{M_{2}+\varepsilon}-b_{22}^{M}\right]-\beta_{2} c_{2}\right\}\left|x_{2}(n)-x_{2}^{*}(n)\right| \\
& -\left\{\beta_{2} e-\beta_{1} d_{2}^{M}\right\}\left|u(n)-u^{*}(n)\right|+\left(\beta_{1} b_{21}^{M}+\beta_{2} c_{1}\right) x_{1}(n) \\
= & -\left\{\beta_{1} A_{22}^{\varepsilon}-\beta_{2} c_{2}\right\}\left|x_{2}(n)-x_{2}^{*}(n)\right| \\
& -\left\{\beta_{2} e-\beta_{1} d_{2}^{M}\right\}\left|u(n)-u^{*}(n)\right|+\left(\beta_{1} b_{21}^{M}+\beta_{2} c_{1}\right) x_{1}(n) \\
\leq & -\delta\left(\left|x_{2}(n)-x_{2}^{*}(n)\right|+\left|u(n)-u^{*}(n)\right|\right)+\rho x_{1}(n),
\end{aligned}
$$

where $\rho=\beta_{1} b_{21}^{M}+\beta_{2} c_{1}$.

Summating both sides of the above inequality from $n^{*}$ to $n$, we have

$$
\begin{aligned}
& \sum_{p=n^{*}}^{n}\left(Q_{1}(p+1)-Q_{1}(p)\right) \\
& \leq-\delta \sum_{p=n^{*}}^{n}\left(\left|x_{2}(p)-x_{2}^{*}(p)\right|+\left|u(p)-u^{*}(p)\right|\right) \\
& \quad+\rho \sum_{p=n^{*}}^{n} x_{1}(p)
\end{aligned}
$$

Hence

$$
\begin{aligned}
& Q_{1}(n+1)+\delta \sum_{p=n^{*}}^{n}\left(\left|x_{2}(p)-x_{2}^{*}(p)\right|+\left|u(p)-u^{*}(p)\right|\right) \\
& \quad \leq Q_{1}\left(n^{*}\right)+\rho \sum_{p=n^{*}}^{n} x_{1}(p) .
\end{aligned}
$$


Then, from (89) and (92) we have

$$
\begin{gathered}
\sum_{p=n^{*}}^{n}\left(\left|x_{2}(p)-x_{2}^{*}(p)\right|+\left|u(p)-u^{*}(p)\right|\right) \\
\leq \frac{Q_{1}\left(n^{*}\right)+\rho \sum_{p=n^{*}}^{n} x_{1}(p)}{\delta}<+\infty .
\end{gathered}
$$

Therefore

$$
\sum_{p=n^{*}}^{\infty}\left(\left|x_{2}(p)-x_{2}^{*}(p)\right|+\left|u(p)-u^{*}(p)\right|\right)<+\infty
$$

which means that

$$
\lim _{n \rightarrow+\infty}\left(\left|x_{2}(n)-x_{2}^{*}(n)\right|+\left|u(n)-u^{*}(n)\right|\right)=0 .
$$

Consequently

$$
\begin{aligned}
& \lim _{n \rightarrow+\infty}\left(x_{2}(n)-x_{2}^{*}(n)\right)=0, \\
& \lim _{n \rightarrow+\infty}\left(u(n)-u^{*}(n)\right)=0 .
\end{aligned}
$$

This completes the proof of Theorem 10 .

Proof of Theorem 11. The proof of Theorem 11 is similar to that of Theorem 10, and we omit the details here.

Remark 12. One of the purposes of this paper is to find out the influence of feedback control variable on the persistent property of the system. Obviously, the answer lies in the relations among conditions $\left(A_{1}\right),(58)$, and (60). Now let us consider conditions $\left(A_{1}\right)$ and $(60)$; there may exist the following subcase:

$$
\frac{b_{11}^{L}}{b_{21}^{M}}>\frac{\exp \left(a_{1}^{M}-1\right)}{a_{2}^{L}}>\frac{a_{1}^{M}}{a_{2}^{L}}>\frac{a_{1}^{L}}{a_{2}^{M}}>\frac{b_{11}^{M} e+d_{1}^{M} c_{1}}{b_{21}^{L} e+d_{2}^{L} c_{1}} .
$$

If $b_{12}^{M} / b_{22}^{L}>d_{1}^{M} / d_{2}^{L}$, then we have

$$
\frac{a_{1}^{L}}{a_{2}^{M}}>\frac{a_{1}^{L}}{\exp \left(a_{2}^{M}-1\right)}>\frac{b_{12}^{M}}{b_{22}^{L}}>\frac{b_{12}^{M} e+d_{1}^{M} c_{2}}{b_{22}^{L} e+d_{2}^{L} c_{2}} .
$$

If $b_{12}^{M} / b_{22}^{L}<d_{1}^{M} / d_{2}^{L}$, then there may exist the following two subcases:

$$
\begin{aligned}
& \frac{a_{1}^{L}}{a_{2}^{M}}>\frac{a_{1}^{L}}{\exp \left(a_{2}^{M}-1\right)}>\frac{b_{12}^{M} e+d_{1}^{M} c_{2}}{b_{22}^{L} e+d_{2}^{L} c_{2}}>\frac{b_{12}^{M}}{b_{22}^{L}} \\
& \frac{a_{1}^{L}}{a_{2}^{M}}>\frac{b_{12}^{M} e+d_{1}^{M} c_{2}}{b_{22}^{L} e+d_{2}^{L} c_{2}}>\frac{a_{1}^{L}}{\exp \left(a_{2}^{M}-1\right)}>\frac{b_{12}^{M}}{b_{22}^{L}} .
\end{aligned}
$$

(101) and (100) (or (102) and (100)) show that conditions $\left(\mathrm{A}_{1}\right)$ and (60) could be satisfied together; that is, for the original permanent system (2), by choosing suitable feedback control variable, species $x_{2}$ will be driven to extinction.

Remark 13. Similarly to the above analysis, if system (2) is permanent, by choosing suitable feedback control variable, the first species $x_{1}$ will be driven to extinction.
Remark 14. From Theorem 7, we can find that feedback control variable has influence on the global attractivity of system (5); that is, only the feedback control variable is very low such that the third inequality of (39) holds; in other words, when inequality $d=\max \left\{d_{1}^{M}, d_{2}^{M}\right\}<\alpha_{3} e^{L} /\left(\alpha_{1}+\alpha_{2}\right)$ holds, the two species can be coexist.

\section{Examples}

In this section we will give three examples to illustrate the feasibility of main results.

Example 1. Consider the following equations:

$$
\begin{aligned}
& x_{1}(n+1)=x_{1}(n) \exp \{0.9+0.1 \sin (\sqrt{2} n) \\
& -(0.25+0.05 \cos (\sqrt{3} n)) x_{1}(n) \\
& -(0.08+0.02 \cos (\sqrt{3} n)) \\
& \times \sum_{s=0}^{\infty} \frac{e-1}{e} e^{-s} x_{2}(n-s) \\
& -(0.45+0.03 \sin (\sqrt{2} n)) \\
& \left.\times \sum_{s=0}^{\infty} \frac{e^{2}-1}{e^{2}} e^{-2 s} u(n-s)\right\}, \\
& x_{2}(n+1)=x_{2}(n) \exp \{0.9+0.1 \sin (\sqrt{2} n) \\
& -(0.6+0.1 \cos (\sqrt{3} n)) x_{2}(n) \\
& -(0.15+0.05 \cos (\sqrt{3} n)) \\
& \times \sum_{s=0}^{\infty} \frac{e-1}{e} e^{-s} x_{1}(n-s) \\
& -(0.42+0.04 \sin (\sqrt{2} n)) \\
& \left.\times \sum_{s=0}^{\infty} \frac{e^{2}-1}{e^{2}} e^{-2 s} u(n-s)\right\}, \\
& u(n+1)=u(n)(1-(0.92+0.03 \cos (\sqrt{3} n))) \\
& +(0.01+0.005 \cos (2 n)) \\
& \times \sum_{s=0}^{\infty} \frac{e^{3}-1}{e^{3}} e^{-3 s} x_{1}(n-s) \\
& +(0.008+0.002 \cos (\sqrt{3} n)) \\
& \times \sum_{s=0}^{\infty} \frac{e^{3}-1}{e^{3}} e^{-3 s} x_{2}(n-s) .
\end{aligned}
$$




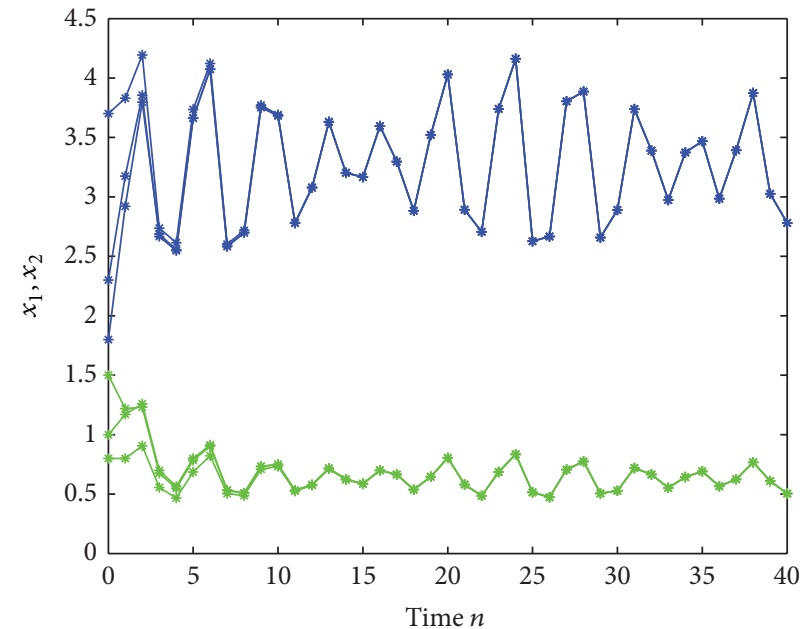

$\rightarrow x_{1}$

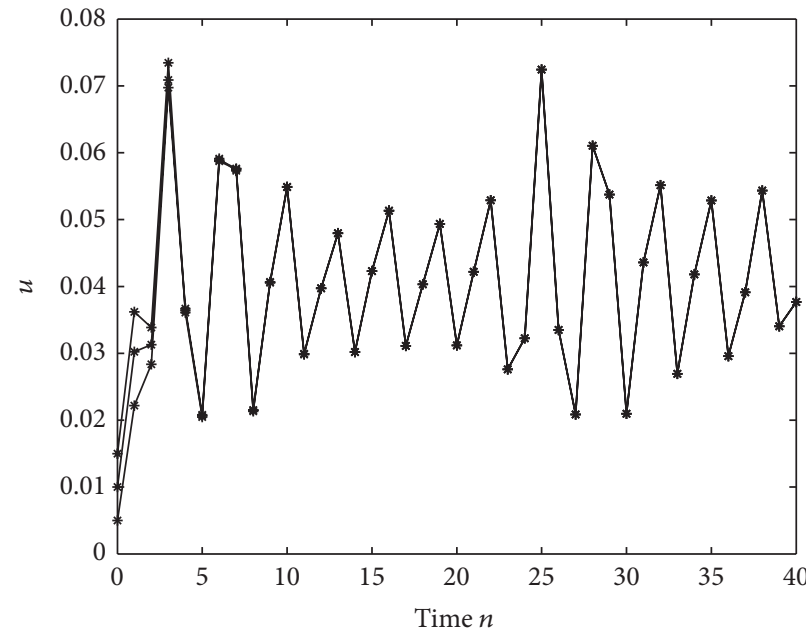

(b)

(a)

FIGURE 1: Numeric simulations of the solutions $\left(x_{1}(n), x_{2}(n), u(n)\right)$ of system $(103)$, with the initial conditions $\left(x_{1}(s), x_{2}(s), u(s)\right)=(1.8,1$, $0.005),(2.3,1.5,0.01)$, and $(3.7,0.8,0.015), s=\cdots,-n,-n+1, \ldots,-1,0$, respectively.

One could easily see that conditions $\left(\mathrm{H}_{1}\right)$ and $\left(\mathrm{H}_{2}\right)$ are satisfied. Also, by calculating, one has

$$
\begin{aligned}
& A_{11}=\min \left\{b_{11}^{L}, \frac{2}{M_{1}}-b_{11}^{M}\right\}=0.1, \\
& A_{22}=\min \left\{b_{22}^{L}, \frac{2}{M_{2}}-b_{22}^{M}\right\}=0.3 .
\end{aligned}
$$

Now, let us take $\alpha_{1}=1.3, \alpha_{2}=0.5$, and $\alpha_{3}=1$; then

$$
\begin{gathered}
\alpha_{1} A_{11}-\alpha_{2} b_{21}^{M}-\alpha_{3} c_{1}^{M}=0.015>0 \\
\alpha_{2} A_{22}-\alpha_{1} b_{12}^{M}-\alpha_{3} c_{2}^{M}=0.01>0 \\
\alpha_{3} e^{L}-\alpha_{1} d_{1}^{M}-\alpha_{2} d_{2}^{M}=0.046>0 .
\end{gathered}
$$

Clearly, condition (39) is satisfied, and so from Theorem 7 we have $\lim _{n \rightarrow+\infty}\left(x_{i}(n)-x_{i}^{*}(n)\right)=0, \lim _{n \rightarrow+\infty}\left(u(n)-u^{*}(n)\right)=$ 0 , where $\left(x_{1}(n), x_{2}(n), u(n)\right)$ and $\left(x_{1}^{*}(n), x_{2}^{*}(n), u^{*}(n)\right)$ are any two positive solutions of system (103).

Figure 1 shows the dynamic behaviors of system (103), which strongly supports the above assertions.

Example 2. Consider the following equations:

$$
\begin{aligned}
x_{1}(n+1)=x_{1}(n) \exp \{0 & .8+0.2 \sin (\sqrt{2} n)-3 x_{1}(n) \\
& -1.5 \sum_{s=0}^{\infty} \frac{e-1}{e} e^{-s} x_{2}(n-s) \\
& \left.-0.1 \sum_{s=0}^{\infty} \frac{e^{2}-1}{e^{2}} e^{-2 s} u(n-s)\right\},
\end{aligned}
$$

$$
\begin{aligned}
x_{2}(n+1)=x_{2}(n) \exp \{1 & 18+0.2 \sin (\sqrt{2} n)-x_{2}(n) \\
& -3 \sum_{s=0}^{\infty} \frac{e-1}{e} e^{-s} x_{1}(n-s) \\
& \left.-0.2 \sum_{s=0}^{\infty} \frac{e^{2}-1}{e^{2}} e^{-2 s} u(n-s)\right\},
\end{aligned}
$$$$
u(n+1)=u(n)(1-0.8)
$$$$
+1.2 \sum_{s=0}^{\infty} \frac{e^{3}-1}{e^{3}} e^{-3 s} x_{1}(n-s)
$$$$
+3 \sum_{s=0}^{\infty} \frac{e^{3}-1}{e^{3}} e^{-3 s} x_{2}(n-s) .
$$

One could easily see that conditions $\left(\mathrm{H}_{1}\right)$ and $\left(\mathrm{H}_{2}\right)$ are satisfied. Also, by calculating, one has

$$
\begin{gathered}
\frac{a_{1}^{M}}{a_{2}^{L}}=\frac{5}{8}, \quad \frac{b_{11}^{L} e+d_{1}^{L} c_{1}}{b_{21}^{M} e+d_{2}^{M} c_{1}}=\frac{9}{10}, \\
\frac{b_{12}^{L} e+d_{1}^{L} c_{2}}{b_{22}^{M} e+d_{2}^{M} c_{2}}=\frac{15}{14} . \\
A_{22}=\min \left\{b_{22}^{L}, \frac{2}{M_{2}}-b_{22}^{M}\right\}=1, \\
0.2=d_{2}^{M}<\frac{A_{22} e}{c_{2}} \approx 0.67 .
\end{gathered}
$$




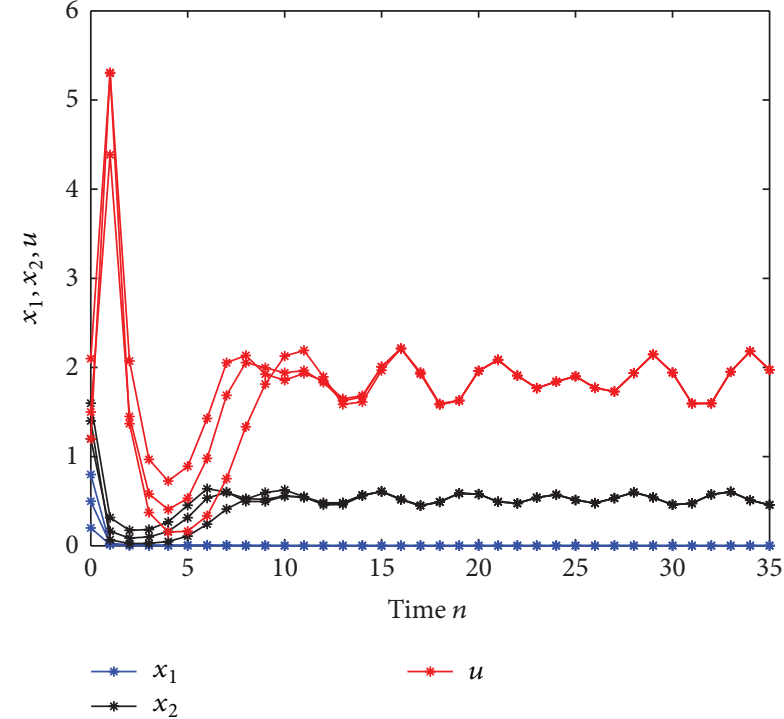

FIGURE 2: Numeric simulations of the solutions $\left(x_{1}(n), x_{2}(n), u(n)\right)$ of system (106), with the initial conditions $\left(x_{1}(s), x_{2}(s), u(s)\right)=$ $(0.8,1.4,1.2),(0.5,1.2,1.5)$, and $(0.2,1.6,2.1), s=\cdots,-n,-n+$ $1, \ldots,-1,0$, respectively.

Clearly, conditions (58) and (81) are satisfied, and so from Theorems 8 and 10 we know that species $x_{1}$ will be driven to extinction, while species $x_{2}$ is globally attractive.

Figure 2 shows the dynamic behaviors of system (106), which strongly supports our results.

Example 3. Consider the following example:

$$
\begin{array}{r}
x_{1}(n+1)=x_{1}(n) \exp \left\{0.9+0.1 \sin (n)-0.6 x_{1}(n)\right. \\
\left.\quad-0.4 \sum_{s=0}^{\infty} \frac{e-1}{e} e^{-s} x_{2}(n-s)\right\}, \\
x_{2}(n+1)=x_{2}(n) \exp \left\{0.8+0.2 \sin (n)-0.8 x_{2}(n)\right. \\
\left.\quad-0.3 \sum_{s=0}^{\infty} \frac{e-1}{e} e^{-s} x_{1}(n-s)\right\} .
\end{array}
$$

By calculating, one has

$$
\begin{gathered}
\frac{b_{11}^{L}}{b_{21}^{M}}=2>\frac{\exp \left(a_{1}^{M}-1\right)}{a_{2}^{L}}=\frac{5}{3}, \\
\frac{a_{1}^{L}}{\exp \left(a_{2}^{M}-1\right)}=0.8>\frac{b_{12}^{M}}{b_{22}^{L}}=0.5 .
\end{gathered}
$$

Clearly, condition $\left(A_{1}\right)$ is satisfied; thus from Theorem A we know that system (108) is permanent. Figure 3 shows the dynamic behaviors of system (108).

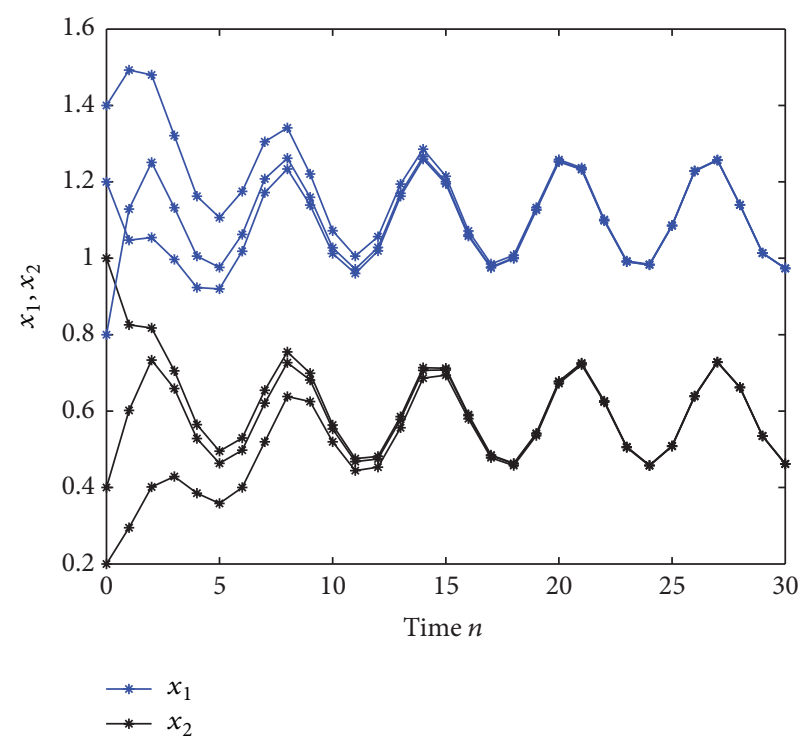

Figure 3: Dynamic behaviors of the solution $\left(x_{1}(n), x_{2}(n)\right)$ of system (110), with the initial conditions $\left(x_{1}(s), x_{2}(s)\right)=(0.8,0.4),(1.4,1.2)$, and $(1.2,1.0), s=\cdots,-n,-n+1, \ldots,-1,0$, respectively.

Now let us further incorporate the feedback control variable into system (108) and consider the following system:

$$
x_{1}(n+1)=x_{1}(n) \exp \left\{0.9+0.1 \sin (n)-0.6 x_{1}(n)\right.
$$

$$
\begin{array}{r}
-0.4 \sum_{s=0}^{\infty} \frac{e-1}{e} e^{-s} x_{2}(n-s) \\
\left.-0.2 \sum_{s=0}^{\infty} \frac{e^{2}-1}{e^{2}} e^{-2 s} u(n-s)\right\}, \\
x_{2}(n+1)=x_{2}(n) \exp \left\{0.8+0.2 \sin (n)-0.8 x_{2}(n)\right. \\
-0.3 \sum_{s=0}^{\infty} \frac{e-1}{e} e^{-s} x_{1}(n-s) \\
\left.-0.3 \sum_{s=0}^{\infty} \frac{e^{2}-1}{e^{2}} e^{-2 s} u(n-s)\right\},
\end{array}
$$

$u(n+1)=u(n)(1-0.5)$

$$
\begin{aligned}
& +5 \sum_{s=0}^{\infty} \frac{e^{3}-1}{e^{3}} e^{-3 s} x_{1}(n-s) \\
& +\sum_{s=0}^{\infty} \frac{e^{3}-1}{e^{3}} e^{-3 s} x_{2}(n-s) .
\end{aligned}
$$




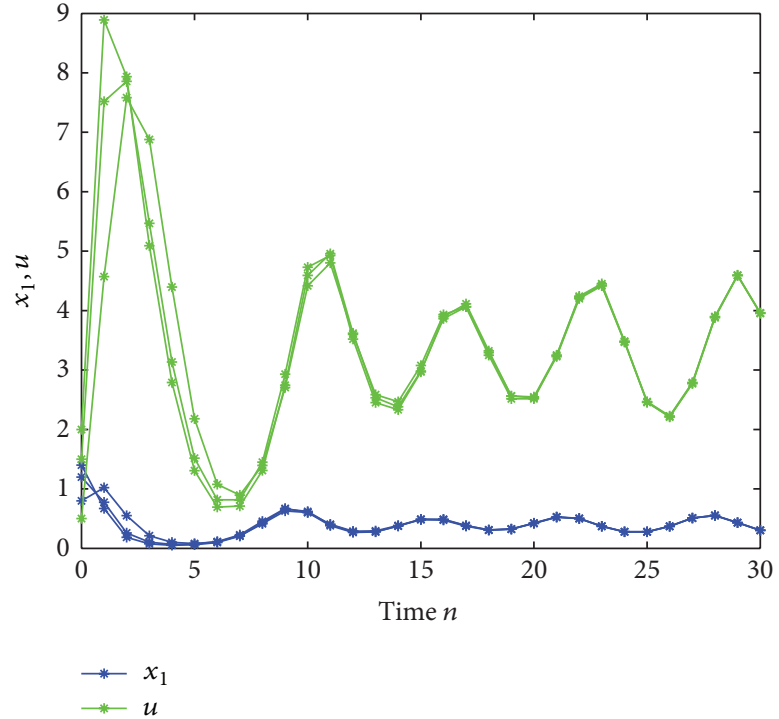

(a)

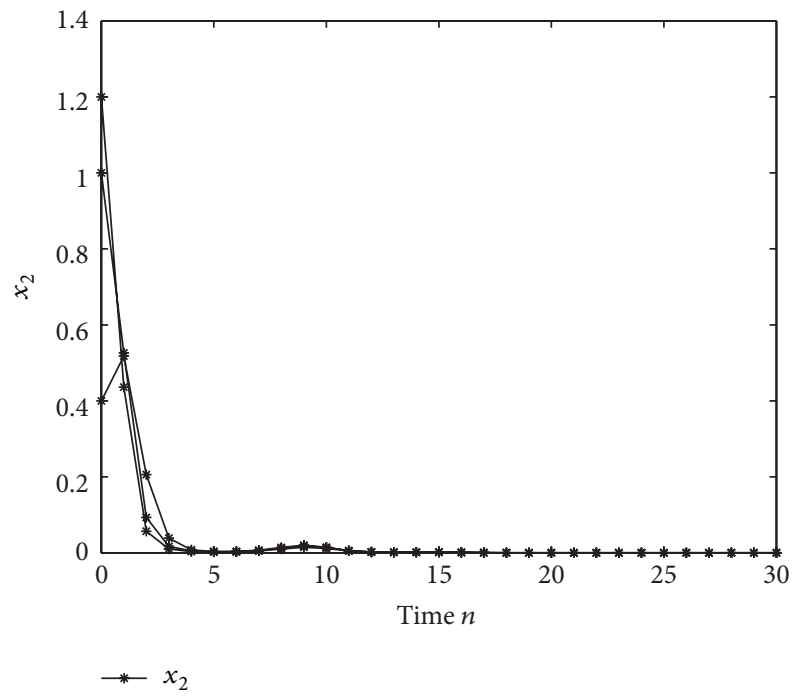

(b)

Figure 4: Numeric simulations of the solutions $\left(x_{1}(n), x_{2}(n), u(n)\right)$ of system $(110)$, with the initial conditions $\left(x_{1}(s), x_{2}(s), u(s)\right)=(0.8,0.4$, $0.5),(1.4,1.2,2)$, and $(1.2,1.0,1.5), s=\cdots,-n,-n+1, \ldots,-1,0$, respectively.

By calculating, one has

$$
\begin{aligned}
& \frac{a_{1}^{L}}{a_{2}^{M}}=\frac{4}{5}>\frac{b_{12}^{M} e+d_{1}^{M} c_{2}}{b_{22}^{L} e+d_{2}^{L} c_{2}}=\frac{4}{7}, \\
& \frac{a_{1}^{L}}{a_{2}^{M}}=\frac{4}{5}>\frac{b_{11}^{M} e+d_{1}^{M} c_{1}}{b_{21}^{L} e+d_{2}^{L} c_{1}}=\frac{26}{33} .
\end{aligned}
$$

Thus, all the conditions of Theorem 9 are satisfied, and so the permanent $x_{2}$ species in system (108) become extinct in system (110).

Figure 4 shows the dynamic behaviors of system (110), which supports our assertion.

\section{Discussion}

(1) Li et al. [3] consider a continuous and autonomous Lotka-Volterra competitive system with infinite delays and feedback controls; if the Lotka-Volterra competitive system is globally stable, then they showed that the feedback controls only change the position of the unique positive equilibrium and retain the stable property. As a consequence of this result, feedback control variables have no influence on the persistent property of the system they considered; $\mathrm{Xu}$ et al. [5] proposed and studied the discrete nonautonomous Lotka-Volterra competitive system with delays and feedback controls (model (4)). Theorem 3.2 in [5] showed that feedback controls do not affect the persistent property of system (4). In this paper, we propose and study a discrete LotkaVolterra competition system with single feedback control variable. Remarks 12 and 13 show that the feedback control variable plays an important role in the persistent property of the system (2). If the Lotka-Volterra competitive system is permanent, then we show that, by choosing suitable feedback control variable, one of the species will be driven to extinction; that is, feedback control variable, which represents the biological control or some harvesting procedure, is an unstable factor of the system. Such a finding overturns the previous recognition on feedback control variables $([3,5])$ and shows that it is better for us to take a single policy to protect the rare species through reducing the species' competitions than to take two different policies.

(2) In [2], Chen proposed system (2) and investigated the persistent property of the system (see Theorem A in introduction section); however, he did not investigate the stability property and extinction property of the system. As a direct corollary of Theorem 7, concerned with the stability property of system (2), we have the following.

Theorem B. Assume that there exist positive constants $\alpha_{i}, i=$ 1,2 , such that

$$
\alpha_{1} A_{11}-\alpha_{2} b_{21}^{M}>0, \quad \alpha_{2} A_{22}-\alpha_{1} b_{12}^{M}>0
$$

hold; then, for any two positive solutions $\left(x_{1}(n), x_{2}(n)\right)$ and $\left(x_{1}^{*}(n), x_{2}^{*}(n)\right)$ of system (2), one has

$$
\lim _{n \rightarrow+\infty}\left(x_{i}(n)-x_{i}^{*}(n)\right)=0,
$$

where

$$
A_{i i}=\min \left\{b_{i i}^{L}, \frac{2}{M_{i}}-b_{i i}^{M}\right\}, \quad i=1,2 .
$$


Now let us consider the autonomous case of system (2). Assume that $a_{i}>1, i=1,2$; then

$$
A_{i i}=\min \left\{b_{i i}, \frac{2 b_{i i}}{\exp \left\{a_{i}-1\right\}}-b_{i i}\right\}=\left(\frac{2}{\exp \left\{a_{i}-1\right\}}-1\right) b_{i i} .
$$

As a corollary of Theorem B, we have the following.

Theorem B' . Assume that $a_{i}>1, i=1,2$, and

$$
\begin{aligned}
& \left(\frac{2}{\exp \left\{a_{1}-1\right\}}-1\right) b_{11}-b_{21}>0, \\
& \left(\frac{2}{\exp \left\{a_{2}-1\right\}}-1\right) b_{22}-b_{12}>0
\end{aligned}
$$

hold; then, for any two positive solutions $\left(x_{1}(n), x_{2}(n)\right)$ and $\left(x_{1}^{*}(n), x_{2}^{*}(n)\right)$ of the system (2), one has

$$
\lim _{n \rightarrow+\infty}\left(x_{i}(n)-x_{i}^{*}(n)\right)=0 .
$$

Remark 15. Wu and Zhang [19] obtained a set of sufficient conditions which ensure the global attractivity of a discrete autonomous Lotka-Volterra competition system with infinite delays and feedback controls; as a direct corollary of their main result, one could also obtain a set of sufficient conditions which ensure the global stability of the autonomous case of system (2). However, their result required the assumption $a_{i} \leq$ $1, i=1,2$. While Theorem $\mathrm{B}^{\prime}$ solves the case $a_{i}>1, i=1,2$. It is in this sense that our result Theorem $\mathrm{B}^{\prime}$ supplements and complements the main result of $\mathrm{Wu}$ and Zhang [19].

Now, concerned with the extinction property of the system (2), with some minor revision of the proof of Theorems 8 and 9 , we could obtain the following results.

Theorem C. Assume that

$$
\frac{a_{1}^{M}}{a_{2}^{L}}<\frac{b_{11}^{L}}{b_{21}^{M}}, \quad \frac{a_{1}^{M}}{a_{2}^{L}}<\frac{b_{12}^{L}}{b_{22}^{M}}
$$

hold; let $\left(x_{1}(n), x_{2}(n)\right)$ be any positive solution of system (2); then

$$
\lim _{n \rightarrow+\infty} x_{1}(n)=0 \text {. }
$$

Theorem D. Assume that

$$
\frac{a_{1}^{L}}{a_{2}^{M}}>\frac{b_{11}^{M}}{b_{21}^{L}}, \quad \frac{a_{1}^{L}}{a_{2}^{M}}>\frac{b_{12}^{M}}{b_{22}^{L}}
$$

hold; let $\left(x_{1}(n), x_{2}(n)\right)$ be any positive solution of system (2); then

$$
\lim _{n \rightarrow+\infty} x_{2}(n)=0 \text {. }
$$

It is interesting to investigate the stability property of the rest of the species when one of the species in system (2) is driven to extinction. In this case, as a direct corollary of Theorems 10 and 11, by simple computation, we have the following.
Theorem E. Assume that (118) holds and also

$$
\frac{b_{22}^{M}}{b_{22}^{L}} \exp \left\{a_{2}^{M}-1\right\}<2
$$

holds; then, for any positive solution $\left(x_{1}(n), x_{2}(n)\right)$ of system (2), we have

$$
\lim _{n \rightarrow+\infty} x_{1}(n)=0, \quad \lim _{n \rightarrow+\infty}\left(x_{2}(n)-x_{2}^{*}(n)\right)=0,
$$

where $x_{2}^{*}(n)$ is any positive solution of the system $x_{2}(n+1)=$ $x_{2}(n) \exp \left(a_{2}(n)-b_{22}(n) x_{2}(n)\right)$.

Theorem F. Assume that (120) holds and also

$$
\frac{b_{11}^{M}}{b_{11}^{L}} \exp \left\{a_{1}^{M}-1\right\}<2
$$

holds; then, for any positive solution $\left(x_{1}(n), x_{2}(n)\right)$ of system (2), we have

$$
\lim _{n \rightarrow+\infty} x_{2}(n)=0, \quad \lim _{n \rightarrow+\infty}\left(x_{1}(n)-x_{1}^{*}(n)\right)=0,
$$

where $x_{1}^{*}(n)$ is any positive solution of the system $x_{1}(n+1)=$ $x_{1}(n) \exp \left(a_{1}(n)-b_{11}(n) x_{1}(n)\right)$.

Obviously, Theorems B-F complement and supplement the main results of Chen [2].

\section{Appendix}

The proof of the solution of system (5) which satisfies the initial conditions (8) is positive.

Proof. For convenience, we introduce the following notations:

$$
\begin{aligned}
F_{i}(n)= & a_{i}(n)-b_{i i}(n) x_{i}(n)-b_{i j}(n) \\
& \times \sum_{s=0}^{\infty} k_{i j}(s) x_{j}(n-s)-d_{i}(n) \sum_{s=0}^{\infty} h_{i}(s) u(n-s), \\
b(n)= & c_{1}(n) \sum_{s=0}^{\infty} l_{1}(s) x_{1}(n-s) \quad i, 2, i \neq j, \\
& +c_{2}(n) \sum_{s=0}^{\infty} l_{2}(s) x_{2}(n-s) .
\end{aligned}
$$

And so, from the first and second equations of system (5), we have

$$
\frac{x_{i}(n+1)}{x_{i}(n)}=\exp \left\{F_{i}(n)\right\}
$$

Therefore

$$
x_{i}(n)=x_{i}(0) \exp \left\{\sum_{p=0}^{n-1} F_{i}(p)\right\}>0 .
$$


From the third equation of system (5), we have

$$
u(n+1) \geq\left(1-e^{u}\right) u(n)+b(n) .
$$

Therefore

$$
u(n) \geq\left(1-e^{u}\right)^{n} u(0)+\sum_{p=0}^{n-1}\left(1-e^{u}\right)^{p} b(n-p-1) .
$$

From conditions $\left(\mathrm{H}_{1}\right)$ and (8), we can know

$$
1-e^{u}>0, \quad b(n-p-1)>0 .
$$

So

$$
u(n)>0
$$

\section{Conflict of Interests}

The authors declare that there is no conflict of interests regarding the publication of this paper.

\section{Acknowledgments}

The research was supported by the Natural Science Foundation of Fujian Province (2013J01011, 2013J01010) and the Foundation of Fujian Education Bureau (JA13361).

\section{References}

[1] Y. Chen and Z. Zhou, "Stable periodic solution of a discrete periodic Lotka-Volterra competition system," Journal of Mathematical Analysis and Applications, vol. 277, no. 1, pp. 358-366, 2003.

[2] F. D. Chen, "Permanence in a discrete Lotka-Volterra competition model with deviating arguments," Nonlinear Analysis: Real World Applications, vol. 9, no. 5, pp. 2150-2155, 2008.

[3] Z. Li, M. H. Han, and F. D. Chen, "Influence of feedback controls on an autonomous Lotka-Volterra competitive system with infinite delays," Nonlinear Analysis: Real World Applications, vol. 14, no. 1, pp. 402-413, 2013.

[4] X. X. Chen and F. D. Chen, "Stable periodic solution of a discrete periodic Lotka-Volterra competition system with a feedback control," Applied Mathematics and Computation, vol. 181, no. 2, pp. 1446-1454, 2006.

[5] J. B. Xu, Z. D. Teng, and H. J. Jiang, "Permanence and global attractivity for discrete nonautonomous two-species LotkaVolterra competitive system with delays and feedback controls," Periodica Mathematica Hungarica, vol. 63, no. 1, pp. 19-45, 2011.

[6] J. L. Liu, J. F. Yu, and J. C. Wu, "Research progress on the effect of chemical pesticides on the plant physiology and biochemistry," Agrocheincals, vol. 48, no. 5, pp. 509-514, 2006 (Chinese).

[7] N. Desneux, A. Decourtye, and J. Delpuech, "The sublethal effects of pesticides on beneficial arthropods," Annual Review of Entomology, vol. 52, pp. 81-106, 2007.

[8] F. X. Yao, H. Zheng, H. Y. Li, and C. Qin, "Effect of chemotherapy on cell immunofunction in patients of lung cancer," Chinese Journal of Clinical Oncology and Rehabilitation, vol. 8, no. 2, pp. 213-218, 2001, (Chinese).
[9] L. Wang and M. Q. Wang, Ordinary Difference Equation, Xinjiang University Press, Xinjiang, China, 1991, (Chinese).

[10] F. D. Chen, "Permanence of a single species discrete model with feedback control and delay," Applied Mathematics Letters, vol. 20, no. 7, pp. 729-733, 2007.

[11] Y. Muroya, "Persistence and global stability for discrete models of nonautonomous Lotka-Volterra type," Journal of Mathematical Analysis and Applications, vol. 273, no. 2, pp. 492-511, 2002.

[12] Y. Muroya, "Persistence and global stability in discrete models of Lotka-Volterra type," Journal of Mathematical Analysis and Applications, vol. 330, no. 1, pp. 24-33, 2007.

[13] L. Zhang, Z. Teng, T. Zhang, and S. Gao, "Extinction in nonautonomous discrete Lotka-Volterra competitive system with pure delays and feedback controls," Discrete Dynamics in Nature and Society, vol. 2009, Article ID 656549, 19 pages, 2009.

[14] G. Y. Chen and Z. D. Teng, "On the stability in a discrete twospecies competition system," Journal of Applied Mathematics and Computing, vol. 38, no. 1-2, pp. 25-39, 2012.

[15] H. Hu, Z. Teng, and S. Gao, "Extinction in nonautonomous Lotka-Volterra competitive system with pure-delays and feedback controls," Nonlinear Analysis: Real World Applications, vol. 10, no. 4, pp. 2508-2520, 2009.

[16] C. L. Shi, Z. Li, and F. D. Chen, "Extinction in a nonautonomous Lotka-Volterra competitive system with infinite delay and feedback controls," Nonlinear Analysis: Real World Applications, vol. 13, no. 5, pp. 2214-2226, 2012.

[17] X. Z. Kong, L. P. Chen, and W. S. Yang, "Note on the persistent property of a discrete Lotka-Volterra competitive system with delays and feedback controls," Advances in Difference Equations, vol. 2, pp. 1-9, 2010.

[18] D. Y. Wu, "Global attractivity of a discrete Lotka-Volterra competition system with infinite delays and feedback controls," Advances in Difference Equations, vol. 2013, article 14, pp. 1-11, 2013.

[19] D. Y. Wu and H. Zhang, "Bifurcation analysis of a two-species competitive discrete model of plankton allelopathy," Advances in Difference Equations, vol. 2014, article 70, 2014.

[20] Q. Y. Li, H. W. Liu, and F. Q. Zhang, "The permanence and extinction of a discrete predator-prey system with time delay and feedback controls," Advances in Difference Equations, vol. 2010, Article ID 738306, 20 pages, 2010.

[21] S. Choo, "Global stability in n-dimensional discrete LotkaVolterra predator-prey models," Advances in Difference Equations, vol. 11, pp. 1-17, 2014.

[22] Z. Yu and Z. Li, "Permanence and global attractivity of a discrete two-prey one-predator model with infinite delay," Discrete Dynamics in Nature and Society, vol. 2009, Article ID 732510, 16 pages, 2009.

[23] L. Chen and X. Xie, "Feedback control variables have no influence on the permanence of a discrete $N$-species cooperation system," Discrete Dynamics in Nature and Society, vol. 2009, Article ID 306425, 10 pages, 2009.

[24] H. Wang, F. Chen, Y. Lin, and W. Chen, "Note on the partial survival of a two species Lotka-Volterra competitive system with infinite delays and feedback controls," Annals of Differential Equations, vol. 29, no. 4, pp. 456-465, 2013.

[25] P. H. Hong and P. X. Weng, "Global attractivity of almostperiodic solution in a model of hematopoiesis with feedback control," Nonlinear Analysis: Real World Applications, vol. 12, no. 4, pp. 2267-2285, 2011. 
[26] H. N. Wang, F. D. Chen, W. L. Chen, and Y. H. Lin, “The permanence and globalattractivity of an non-autonomous two species allelopathic phytoplankton model with feedback controls," Journal of Shenyang University, vol. 25, no. 3, pp. 173-176, 2013 (Chinese).

[27] Y. Fan and W. Li, "Permanence for a delayed discrete ratiodependent predator-prey system with Holling type functional response," Journal of Mathematical Analysis and Applications, vol. 299, no. 2, pp. 357-374, 2004.

[28] F. D. Chen, "Average conditions for permanence and extinction in nonautonomous Gilpin-Ayala competition model," Nonlinear Analysis: Real World Applications, vol. 7, no. 4, pp. 895-915, 2006.

[29] X. Chen and F. Chen, "Periodicity and stability of a discrete time periodic $n$-species Lotka-Volterra competition system with feedback controls and deviating arguments," Soochow Journal of Mathematics, vol. 32, no. 3, pp. 343-368, 2006.

[30] X. Y. Liao, Z. G. Ouyang, and S. Zhou, "Permanence of species in nonautonomous discrete Lotka-Volterra competitive system with delays and feedback controls," Journal of Computational and Applied Mathematics, vol. 211, no. 1, pp. 1-10, 2008. 


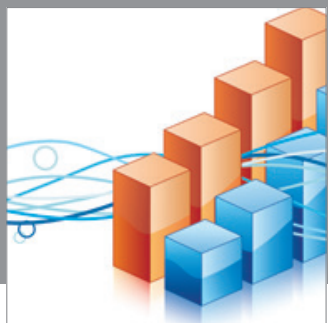

Advances in

Operations Research

mansans

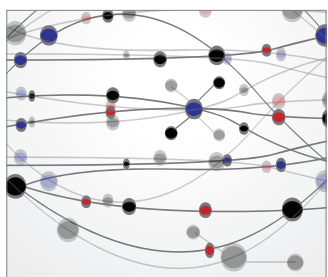

The Scientific World Journal
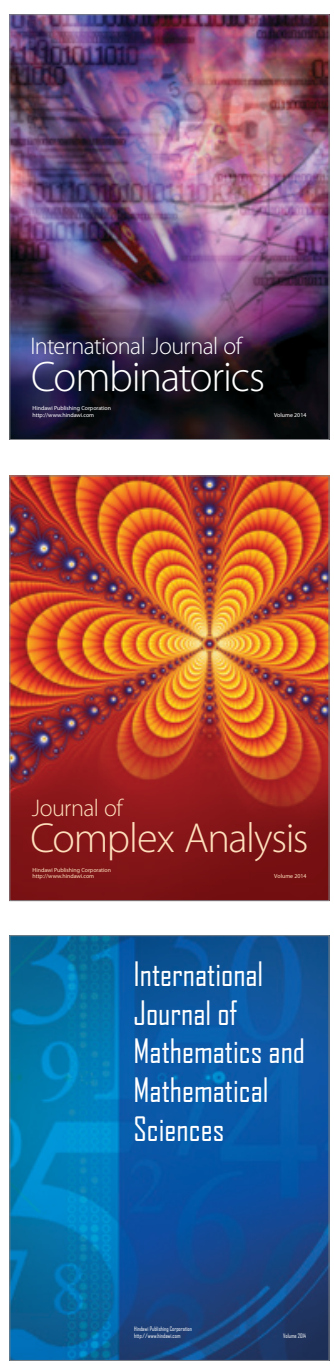
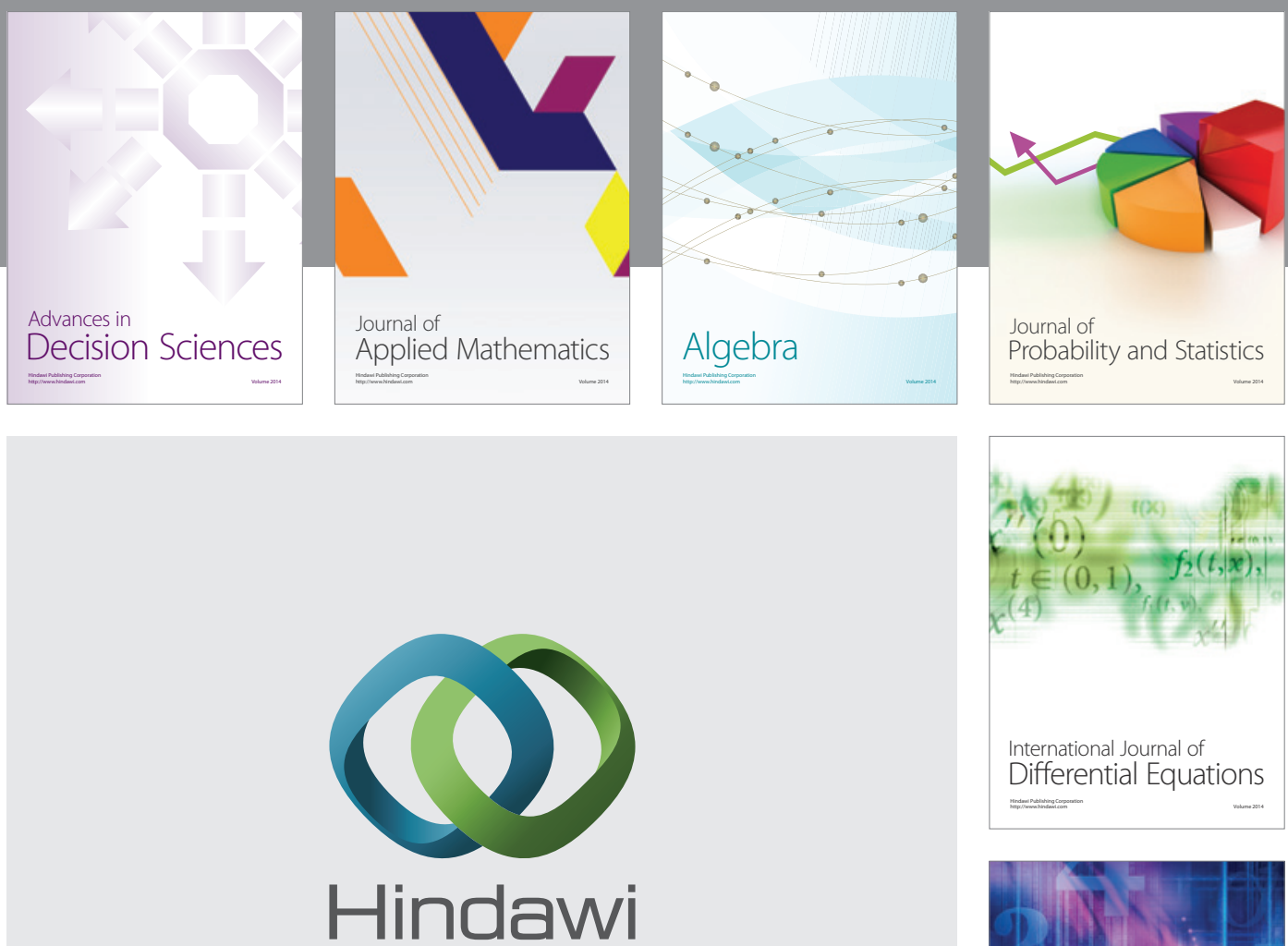

Submit your manuscripts at http://www.hindawi.com
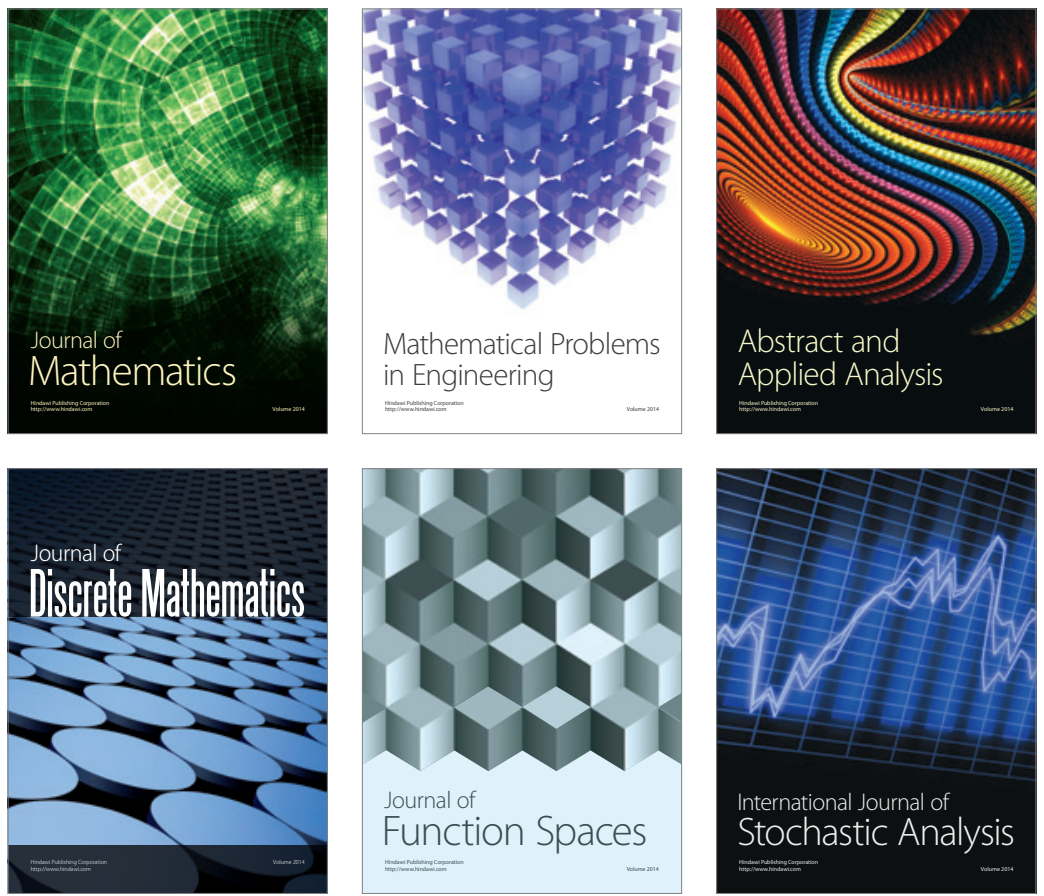

Journal of

Function Spaces

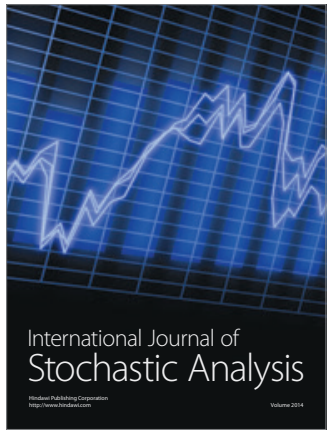

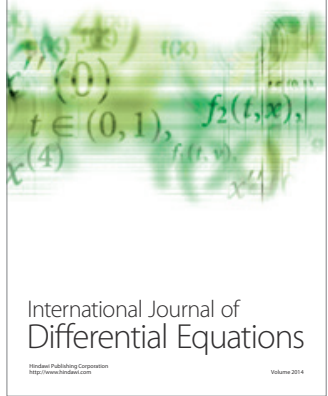
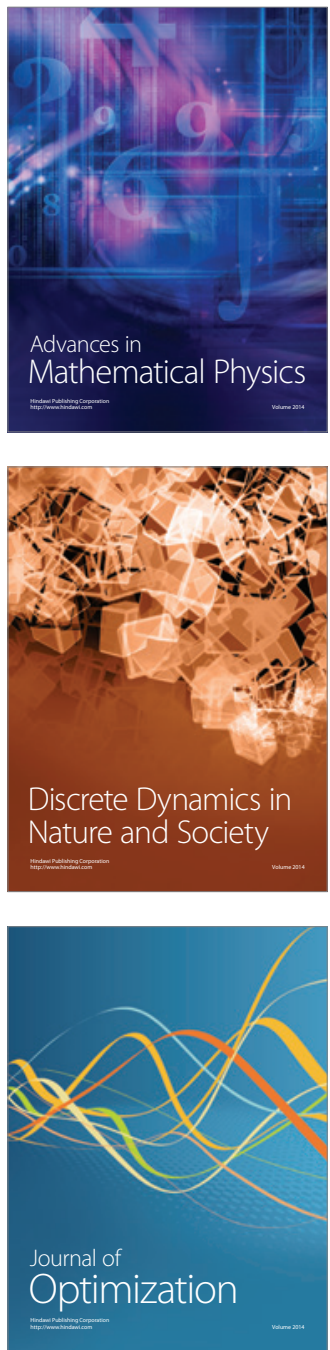